NATL INST OF STANDARDS \& TECH R.I.C.

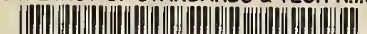

A11102753748

NBSIR $87-3077$

\title{
TIME-DOMAIN SYSTEM FOR
} IDENTIFICATION OF THE NATURAL RESONANT FREQUENCIES OF AIRCRAFT RELEVANT TO ELECTROMAGNETIC COMPATIBILITY TESTING

John W. Adams

Arthur R. Ondrejka

Herbert W. Medley

National Bureau of Standards

U.S. Department of Commerce

Boulder, Colorado 80303-3328

November 1987

$Q C$

100

.456

87-3077

1987 



\section{TIME-DOMAIN SYSTEM FOR}

\section{IDENTIFICATION OF THE NATURAL}

RESONANT FREQUENCIES OF AIRCRAFT

RELEVANT TO ELECTROMAGNETIC

John W. Adams

Arthur R. Ondrejka

Herbert W. Medley

Electromagnetic Fields Division

Center for Electron'c and Electrical Engineering

National Engineering Laboratory

National Bureau of Standards

Boulder, Colorado 80303-3328

November 1987

Sponsored by

U.S. Army Aviation Systems Command

St. Louis, Missouri 63120

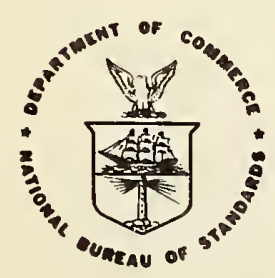

U.S. DEPARTMENT OF COMMERCE, C. William Verity, Secretary 
CONTENTS

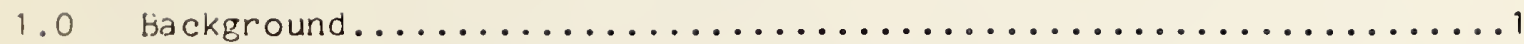

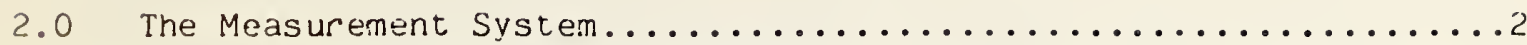

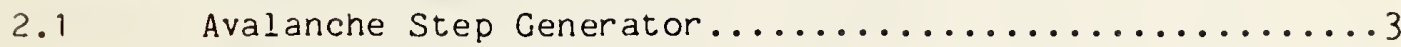

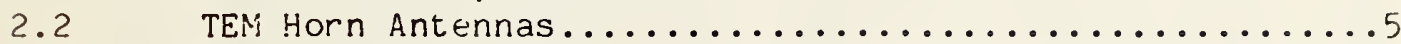

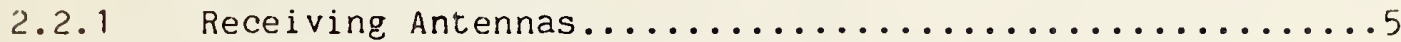

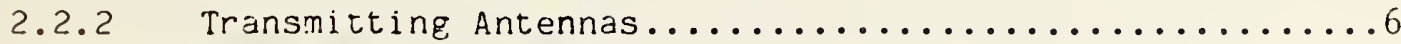

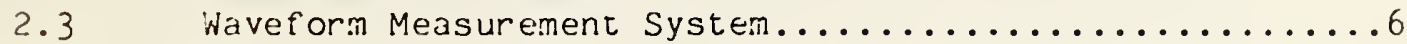

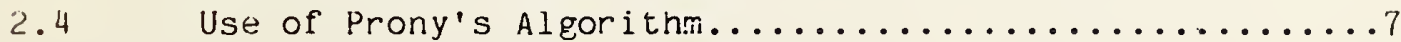

3.0 Measurement Procedure Used at Ft. Rucker................

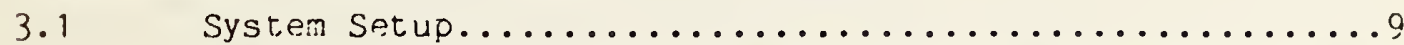

3.2 Selecting Appropriate Parameters for Time-Domain System.9

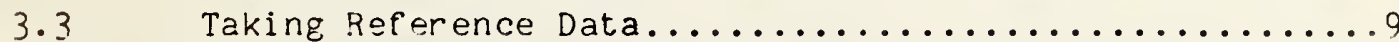

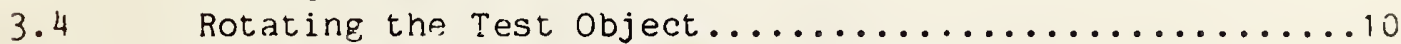

3.5 Filtering, Analog Data Before Digitizing............

3.6 Processing and Interpreting the Data.............

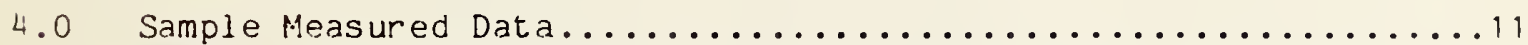

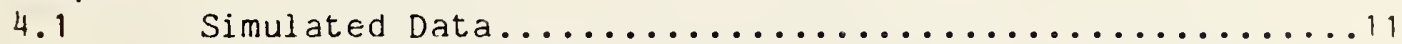

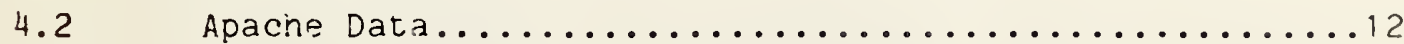

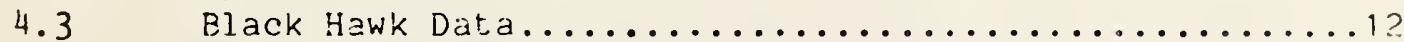

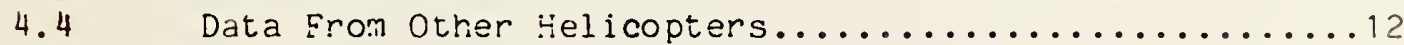

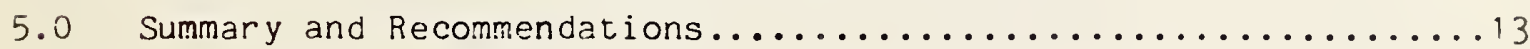

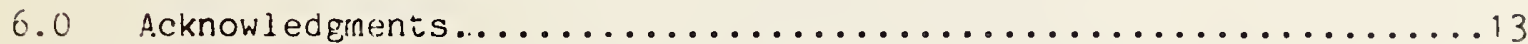

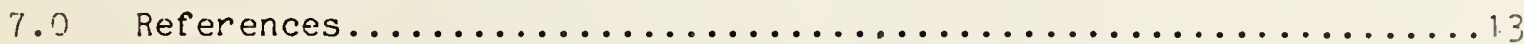



Time-Domain System for Identification of the Natural Resonant

Frequencies of Aircraft Relevant to Electromagnetic Compatibility Testing

John W. Adams, Arthur R. Ondrejka and Herbert W. Medley

A method of measuring the natural resonant frequencies of a structure is described. The measurement involves irradiating this structure, in this case a helicopter, with an impulsive electromagnetic (EM) field and receiving the echo reflected from the helicopter. Resonances are identified by using a mathematical algorithm based on Prony's method to operate on the digitized reflected signal. The measurement system consists of special TEM horns, pulse generators, a time-domain system, and Prony's algorithm. The frequency range covered is 5 megahertz to 250 megahertz; this range is determined by antenna and circuit characteristics.

The measurement system is demonstrated, and measured data from several different helicopters are presented in different forms. These different forms are needed to determine which of the resonant frequencies are real and which are false. The false frequencies are byproducts of Prony's algorithm.

Key words: avalanche step generator; natural resonant frequencies; Prony's method; TEM horn antennas; time-domain measurement system.

\subsection{Background}

If a structure is exposed to electromagnetic fields, resonances enhance the fields by a factor of $Q$ and often cause electromagnetic compatibility (EMC) problems. Structures such as helicopters are built of highly conductive materials such as aluminum, which typically yield Qs of 10 to 200. The quality factor, $Q$, is the ratio of stored to dissipated energy. If the shape of a structure is complicated, as is the case with helicopters, these resonant frequencies cannot be calculated. The only way to obtain these resonances is by measurements. Fortunately, advances in antenna design and solid state technology can be used with existing mathematical procedures to develop a measurement system that can make the needed measurements.

A technological advance that will change some of the aircraft characteristics is the increasing use of composite materials in place of aluminum. For lossless structures, size-frequency scaling might be used for the type of measurements discliss in this paper, but for lossy structures, measurements should be made on full-size aircraft rather than scale models, since size-frequency scaling, does not work effectively for lossy objects.

Prony's algorithm has been in existence for about 200 years. More recent applications to determine poles from time-domain waveforms have met with varying degrees of success [1-5]. As a tool for target identification, it has only limited success, since the quality of results degrades as the 
signal-to-noise ratio decreases. If the target is not stationary with respect to a transmitting antenna, only one time interval of data may be recorded. However, if the position of a test object is fixed relative to transmitting and receiving antennas, a large number of time intervals may be recorded and averaged, which gives a significant improvement in signal-to noise ratio.

Time-domain technology has been more easily handled in theory than in application, but its great potential can now be realized due to the recent improvements in the electronics and antennas needed for its application. Higher speed devices such as gallium arsenide transistors [6-7] are one area where significant improvements are resulting in faster and more powerful impulse generation capability. Antennas that radiate impulses without significant phase distortion are fairly straightforward to construct, and the needed theory concerning differences between transmitting and receiving horn antennas is now available [8]. These transverse electromagnetic (TEM) horn antennas have been built and evaluated previously, but only for higher frequency ranges. For EMC testing, the lower frequency ranges typical of resonances of larger structures had not been covered.

Electromagnetic compatibility (EMC) testing for incident fields should be performed at all frequencies to which the device or system may be exposed. The lower portion of the spectrum where large structures may be resonant is often not tested. This is because common test facilities have severe limitations which preclude their use in these lower frequencies. Anechoic rooms have increasingly high reflections at lower frequencies. TEM cells or parallel plate structures can be used at low frequencies, but have practical limitations for size of test object. Outdoor ranges cannot be used for swept frequency testing, since use of field strength levels high enough for immunity tests would not comply with FCC regulations. Even for EMC testing under these constraints, the testing can be much more effective if the resonant frequencies of the structure are known. Finding these frequencies is the purpose of the work reported here.

\subsection{The Measurement System}

The measurement system used at Ft. Rucker on several Army helicopters is shown in figure 1. The aircraft under test (AUT), whose resonances are being measured, is located on a ground screen which is continuous from transmitting antenna to receiving antenna. The transmitting antenna launches an impulsive field toward the AUT, and properly polarized reflections or reradiations are detected at the receiving antenna. The two antennas are oriented orthogonally to each other to reduce the direct coupling of energy between them. The AUT was in its flight configuration, with no tow bars connected or doors or panels open, but in a power-down condition.

The actual resonant frequencies are unknown, so that it is most efficient to illuminate a target with a spectrum that contains as many different frequencies as possible. The impulse waveform has a spectrum which contains a broad range of frequencies. The spectrum of other waveforms usually favors a narrower band of frequencies. 
Time-domain antenna theory shows that an antenna's characteristic in the transmitting mode is the time derivative of the receiving mode response [8]. To produce a radiated impulse field, it is necessary to drive the transmitting antenna with a step waveform.

The outgoing impulse is produced by a step function followed by a slow ramp which returns the voltage to its original value. The waveform is repetitive at a rate determined by the clock generator. The trigger pulse and $d c$ power are fed to the step generator through a combiner.

The receiving antenna output can be viewed on an oscilloscope, which also serves as a waveform digitizer. For better control of the scope's sweep delay, the scope was synchronized externally to the clock generator. The computer was programmed to record the digitized data so it could be transmitted to a mainframe computer for further analysis at a later time.

\subsection{Avalanche Step Generator}

The step generators used on this project were of the avalanche type. After testing many transistors for maximum applied voltage before avalanche, consistency of avalanche voltage, and switching speed, we used a 2 N3904 npn transistor in a single stage for the preliminary test. In the final configuration, we increased the number of stages from one to four to increase the output amplitude by about a factor of four. This was necessary due to the low amplitude of the differentiated output pulse. The 2 N3904 transistor switches slowly enough in the avalanche mode that a substantial amount of the applied voltage is lost before the pulse can be formed. This is due in part to the antenna's discharging before the transistor can be fully switched on. Also some of the applied voltage is lost due a voltagedivider effect when the pulse passes impedance-matching resistors at the input of the antenna. These resistors are necessary to prevent multiple reflections if part of the pulse is reflected from the mouth of the antenna. These effects caused the actual output pulse to be only 3 to 4 volts in amplitude at the mouth of the horn and 1 to 2 nanoseconds in duration at the transmitting antenna, and the received pulse with this configuration was only 30 to 40 millivolts in amplitude.

In subsequent testing, we found an experimental transistor that showed signs of being much faster in the avalanche mode at substantially lower applied voltages than others tested. That this transistor would avalanche at a lower voltage is a convenience, but the faster avalanche time gives a much faster rise time as a step-function generator. This transistor has a part number HXTR-3615. Another transistor, the HXTR-5104, which is even faster, operates at 40 to 100 volts dc with specified switching speeds of 20 to 80 picoseconds. In the single stage version, this transistor achieved an output pulse of 450 picoseconds with an amplitude of 40 volts. The switching times are greatly reduced by shortening lead lengths and by selective screening of components.

The step generator circuit shown in figure 2 is similar to the one that drives the transmitting antenna, but can be modified to operate without the antenna attached. The combined dc high voltage and a 5-volt positive 
trigger pulse are applied to the input. The dc voltage charges the 220 picofarad capacitor to a voltage just a little less than that needed to avalanche the transistor. The trigger pulse coupled to the transistor base through the 1000-picofarad capacitor initiates the avalanche with very little time jitter. For test purposes, the step output is taken across the 50-ohm resistor at the output. In actual use, the 220-picofarad capacitor is replaced with the antenna which has about the same capacitance, and the 50ohm output resistor is grounded.

During the tests performed at Ft. Rucker, we ran into several problems due to the step generators, grounding, and the ground screen itself:

1. Without proper grounding of the step generators, the pulse would radiate along the transmitting trigger cable and ground screen.

2. The ground screen was made of chicken wire, wired together and staked to the ground. With wind, temperature and humidity changes, the ground screen would shift relative to other physical components, causing changes to the shape of the radiated pulse.

3. To achieve the relatively high dc voltages necessary for operation of the step generators and to obtain a clean supply voltage, we used ten 67.5 volt dry cells in series. Even though the step generators drew very little current, a few microamperes, the temperature and humidity changes caused the available voltage to drop below the operating range of the step generators under some conditions.

To solve these problems we have done several additional tests and suggest the following improvements for any subsequent tests:

1. Work has been done on the component selection and mechanical design of the step generators. After testing the various transistors, we have chosen two versions for a monolithic chip. Using these and selectively choosing them for specific switching and avalanche characteristics, we can have them assembled on a substrate by a hybrid IC manufacturer. By using hybrids assembled in a machined brass package with male and female SMA connectors, we can achieve a small, $1.27 \times 1.91 \times 0.64$ centimeter $(0.5 \times$ $0.75 \times 0.25$ inch), shielded package that can be bolted to the ground screen, thus solving our grounding problem. The step generator can be optically triggered, further reducing cable radiation.

2. In the immediate vicinity of the antennas we would construct 1 rigid ground screen from three sections of 0.91 by 1.22 meter, 0.16 centimeter thick printed circuit board material with 2 ounce copper per square foot (28.35 grams per 0.0929 square meters) on one side. We would join these with 10.16 centimeter wide, adhesive copper foil tape. This would give a 0.91 by 3.66 meter rigid mounting surface for the step generators and antennas. This would stop the movement of the antenna relative to the ground screen.

3. Although the new step generator design allows much lower operating voltages, we feel that a well regulated and filtered power supply would be the best choice to power the step generators. We have located a power 
supply that delivers 600 volts de at 1.5 amperes and has remote current and voltage sense features. It is also IEEE 488 bus controllable. This source would also eliminate downward dc voltage drift typical of batteries being drained by use.

All of the above equipment works well with the half horn antennas which are suitable for radiating pulses with vertically polarized $E$ fields, but the full-horn antennas needed for radiating horizontally polarized E fields suffered signal loss due to ground loading when they were in close proximity to the ground screen. To reduce this effect, tripod supported stands to raise the antennas 3.7 to 4.6 meters above the ground are needed. These stands would be adjustable from the ground in both the horizontal and vertical planes so the antennas could be focused on the test object.

Tests have been performed with these new step generator designs on both the indoor and outdoor ground screens at NBS and the results are very promising.

\subsection{TEM Horn Antennas}

The antennas used for these measurements have to transmit and receive impulsive waveforms with minimum distortion. They were developed from a basic TEM horn design described by Lawton and Ondrejka [8]. In the receiving mode they have a usable bandwidth from 5 megahertz to 1 gigahertz uver which the response is flat within \pm 6 decibels.

The antenna is constructed in two forms, a hal $\mathrm{f}$ horn that is mounted directly onto a ground plane, and a balanced horn that can be used independently of a ground plane. The half horns are useful when operating with vertically polarized fields; the balanced horns can be used with arbitrarily linearly polarized EM fields. Most of the measurements at Ft. Rucker were made using the half-horn configuration.

\subsubsection{Receiving Antennas}

Figure 3 shows the construction of the balanced receiving antenna. The two triangular conductors form a balanced 200-ohm transmission line, a simple modification of the parallel plate transmission line. The spacing between the conductors tapers to a point where they connect to the 200-ohm input of a broadband pulse transformer. This terminates the antenna in its characteristic impedance, which minimizes reflections. The output of the transformer is unbalanced 50 ohms, which matches the coaxial cable we used. The upper frequency 1 imit is about 1.2 gigahertz due to the transformer. The lower frequency limit is about 5 megahertz due to the antenna.

When the antenna is mounted on the ground plane, only half of this structure is needed. The second half appears as an image in the ground plane. Therefore, we refer to this as the half-horn antenna. For convenience, the antenna is constructed to have a 50 -ohm impedance so that the antenna can directly feed a 50-ohm measurement system. The upper frequency limit of the antenna is 3.0 gigahertz, while the lower frequency limit is 5 megahertz. 
Other antennas are relatively broadband but of ten do not have good phase response. With excessive phase shift, an antenna disperses a pulse by delaying different frequency components by differing amounts. This phase distortion makes it difficult to perform radiated time-domain measurements and obtain meaningful results. The NBS antennas have a linear phase response over the passband, making possible the acquisition of a high fidelity waveforms.

\subsubsection{Transmitting Antennas}

The transmitting antennas are the same as the receiving antennas except that a transformer was not used. Instead, the antenna elements were connected directly to the step generator described in a previous section. The antenna itself is the pulse-forming capacitor for the step generator and is charged to the high dc voltage needed for breakdown.

Figure 4 shows the half-horn antenna including a step generator. When the step waveform propagates to the aperture, the antenna produces two types of fields, a step-like function in the near field, and an impulse function in the far field. Only the impulse radiates at a significant distance from the antennas.

Not all the dc energy stored in the antenna is radiated. Some is reflected from the aperture and propagates back to the step generator which remains in a state of avalanche. The resistor $R 1$ in figure 2, located between the transistor collector and the antenna, matches the antenna impedance so that multiple reflections are prevented. Figure 5 shows the difference in radiated waveform for various values of $R 1$. There are very few reflections in the waveform from the matched generator compared to the unmatched one, and most of these are external to the antenna. However, the peak amplitude in the matched condition is half that of the unmatched condition since the matching termination for the source requires half the voltage.

Because the received signal is always contaminated by noise, it is desirable to increase the amplitude of the transmitted signal as much as possible. A modified circuit, shown dashed in figure 2 , introduces a diode shunting $R 1$ to enhance the output step while still retaining the 200 -ohm load resistor for reflections. The amplitude of the resulting pulse with the diode is 50 percent larger than the matched pulse without the diode. No additional reflections are produced by the diode.

\subsection{Waveform Measurement System}

Signals from the antennas are measured directly on a digitizing oscilloscope. The input plug in unit to the oscilloscope is capable of sampling waveforms as fast as 3 nanoseconds, with an equivalent bandwidth in excess of 200 megahertz. At these frequencies, the oscilloscope has to be operated in the repetitive sampling mode. The scope is capable of multiple acquisitions and can calculate an average waveform. It took about a minute to acquire an average of 1000 waveforms the way the scope was set for use at Ft. Rucker. This amount of averaging enhanced the signal-to-noise ratio by about a factor of 30 . 
The digitized information was also transferred to a personal computer via the IEEE-488 bus where it could be displayed or transformed to the frequency domain. After this optional processing of the waveform, the digitized data file was stored on a floppy disk for additional processing using more powerful algorithms on a mainframe computer. The programs needed in the personal computer included a data transfer routine to establish communication between the computer and the oscilloscope, a program that could calculate the fast Fourier transform (FFT) and graph both the timedomain waveform and the its spectrum, a file conversion routine that could change the data file from that which was generated by the oscilloscope to a form that could be transferred to a mainframe computer, and a program which could convert data from the mainframe computer into a form suitable for graphic displays.

\subsection{Use of Prony's Algorithm}

Prony's algorithm is a mathematical procedure to extract frequencies and corresponding residues from a time-domain waveform which contains damped sinusoids, as illustrated in figure 6. After the input data is processed, the output data is given as frequencies and residues in the $\mathrm{s}$ plane. The frequencies appear as conjugate pole-pairs in the second and third quadrants of the complex s plane; only the second quadrant poles are shown in subsequent figures, with radians converted to hertz.

The number of pole pairs is usually unknown. Since one of the inputs needed for Prony's algorithm, in addition to the time-domain waveform, is the number of poles, we had to experiment with known waveforms and try requests for a different numbers of poles. We found no satisfactory explanation or guidance in the literature. A heuristic guideline that we came up with works consistently, but for the mathematical purists, nothing short of a rigorous proof is convincing. In any case, additional theoretical work might be appropriate to evaluate the procedure we developed by trial and error on known waveforms. This procedure is explained as follows.

Repeated runs requesting different numbers of poles from Prony's algorithm for each data set are a key to this procedure. The number of poles is not known beforehand. If there are $n$ true poles and $m$ poles are requested where $m<n$, Prony's algorithm breaks down completely and gives randomly located false poles that are not even close to the true poles. If $m$ poles are requested, where $m>n$, then $n$ poles are correctly located, while $m-n$ poles are randomly located. If $p$ poles are requested where $p>$ $n$, and $p \neq m$, $n$ poles are again correctly located, and $p-n$ poles are randomly located. Repeating this request about four times seems to be sufficient to tell which poles have identical locations and can therefore be identified as true poles. The randomly located poles only overlap by chance, and in the two-dimensional s (complex frequency) plane, this seldom occurs, certainly not for all four requests.

These conditions are shown in four figures produced from a generaten curve with 25 poles from 7 to $246 \mathrm{MHz}$. If $\mathrm{m}$ or $\mathrm{p}$ are greater than 50 (for 25 complex conjugate pole pairs), the 25 frequencies are shown correctly in figures 7 and 8 by the repeated poles that form a tilted $z$. In rigure 9 , 
the solutions from request of 55 poles ( $x^{\prime} s$ ) and request of 50 poles ( $\nabla^{\prime} s$ ) are correct; the solutions from request of 45 poles (非's) are close in some cases but substantially in error other cases; the solutions from request of 40 poles is grossly in error everywhere. Figure 10 shows the meaningless scatter obtained when all requests were for less than 50 poles.

Although plots can give this information, it is much clearer to watch a buildup of the poles on a CRT display in order to separate actual poles from artificial poles. For example, it is hard to distinguish between two, three or four poles printed at the same location. For this we used a personal computer with a high resolution video display.

We used this procedure and Prony's algorithm to determine roots of these digitized time-domain waveforms. We generated three different formats for each set of output data. These three formats are (1) a plot of the poles in the second quadrant of the s plane, (2) a bar plot showing the magnitude of the complex residues of the poles and (3) a bar plot of the Qs of the poles.

The pole plot in the $s$ plane shows the approximate natural resonant frequency of a pole (proportional to the $j \omega$ value) and the damping factor of each pole (related to the $\sigma$ value). Poles near the frequency axis indicate slowly damped sinusoids and will have high Qs. Poles further to the left indicate more rapidly damped sinusoids and will be of little concern since their $Q$ values are low.

The bar plot of the magnitude of the complex residues shows their amplitudes. The amplitude is related to the power of the frequency source for the particular pole and is therefore related not only to the gain of both antennas of our measurement system but also to the transmitting and receiving characteristics of the AUT. The residues may also be used several ways to assist in the separation of true poles from artificial poles. The magnitude of the complex residue is one dimensional, so random repetitions have a better chance of occurring than in the two-dimensional s-plane plots above. The residues repeat for the four runs for the lower frequency poles only if the algorithm has a true pole. The residues of the artificial poles will not repeat unless the magnitude is extremely low. A very low residue is typical of poles extracted from system noise. These show a very high $Q$ and may be fairly tightly clustered. The poles with low-residues appear to be repetitive because visual displays of very small numbers seem about equal. The high residues typical of poles in the lower frequency range are not repeated for all four runs and are therefore relatively easy to use in identifying true or false poles.

The $Q$ of each pole is given by the ratio of imaginary and real parts of the complex pole. For EMC applications this is a very important parameter since it gives an estimate of how much unperturbed fields may be enhanced by the resonance of the structure.

\subsection{Measurement Procedure Used at Ft. Rucker}

There are several steps to obtain the final answers. First, the timedomain system and transmitting and receiving antennas must be set up in a 
way that minimizes cross coupling and reflected signals from unwanted objects, and that maximizes desired signals from the AUT. Second, the timedomain parameters such as the sampling rate must be selected appropriately for the preceding placement. Third, reference signals must be recorded with no test object present so that this signal can be subtracted from signals with test object present in order to remove the effects of reflections and some time-domain system characteristics. Fourth, signals must be obtained for several orientations of the AUT since coupling varies depending on the antenna pattern of the test object and the two measurement system antennas. Fifth, the filtered and digitized data must be recorded on disks. Sixth, these digitized data must be processed using Prony's method and the output data must be displayed in several different forms in order to obtain the correct, interpretation.

\subsection{System setup}

The transmitting and receiving antennas were set up on suitable ground screens with a $90^{\circ}$ angle between them, with both aimed at a center point as shown in figure 1. Typical distances are shown but some adjustments were necessary depending on the size of the AUT. It was necessary to use a dummy target during setup to obtain large enough received signal to make the initial setup of the measurement system.

\subsection{Selecting Appropriate Parameters for Time-Domain System}

The sampling rate, the time between samples and the number of samples to be used for averaging were determined based on size of test object, separation distances, received signal strength, and desired frequency range to be covered. The most commonly used values at $F t$. Rucker were the following. The clock frequency was $10 \mathrm{kilohertz,}$ the sampling density was set for 512 points per waveform, and the scope sweep speed to 100 nanoseconds per division. These settings gave a 1.953 nanosecond time interval between samples.

The clock frequency was chosen to be high enough to allow acquisition of a data set in less than one minute, but low enough that the averaze current does not degrade the avalanche transistor due to overheating. The scope sweep speed was chosen to capture all of the reflections from the aircraft. The 512 points per waveform gives very good time resolution of the reflected waveform.

\subsection{Taking Reference Data}

A reference run with no test object present was made at the beginning and end of each data recording session. In addition to the measurement system characteristics that need to be removed later by subtracting this reference data, there were many other external signals that were removed both by this subtraction and by averaging. The strongest, signals came from the air-to-ground and ground-to-air radio communications. These would literally cover the screen of the oscilloscope at times. There were some fi: stations and other signals of unknown orlgin. 


\subsection{Rotating the Test Object}

The AUT was rotated through $45^{\circ}$ increments for a total of eight orientations. The natural resonant frequencies of the AUT do not change with this rotation, but the coupling of energy in and out of the AUT would vary depending on the patterns of the antenna formed by the AUT and by our transmitting and our receiving antennas. If any one orientation couples a very small amount of energy because of a null in an antenna pattern, important information might have been lost. The optimum number of orientations needed are not known due to the structural complexity of the AUT. The eight rotations we used seemed to be adequate.

For each rotation of the AUT, requests for four different numbers of poles $(99,98,97$ and 96) were made of Prony's algorithm. Data from a single rotation is shown in the illustrations.

\subsection{Filtering Analog Data Before Digitizing}

Analog data must be filtered before it is digitized to prevent aliasing [1]. Using a low-pass filter with sharp roll-off starting at least an octave below Nyquist's folding frequency will prevent signals at higher frequencies from being imaged downward about this folding frequency and contaminating the lower-frequency data. Once the data are digitized, there is no way to remove these imaged frequencies or to tell which are imaged and which are true frequencies.

We did not pay sufficient attention to this problem. One of the plugin units for the digitizing oscilloscope with built-in filtering was damaged in shipment; a back-up, lower frequency plug-in unit only had minimal filtering, and we did not have the proper filters along to use as backup. Therefore, although the data illustrate the ability of the measurement system to function as intended, the data may possibly be contaminated by aliased frequency poles. This aliasing would not cause frequency-shift errors in the poles we did detect, nor would it cause true poles to be missed. Any critical use of these data should be verified by repeating the measurements and including sufficient filtering.

\subsection{Processing and Interpreting the Data}

The digitized data were then stored on disk for later processing on a mainframe computer. Some reformatting of the data was necessary before they were sent to a mainframe computer. We used a personal computer for this task. We also used this computer and a modem to transfer the formatted data to a mainframe computer and also to retrieve the processed results. Again, additional formatting was necessary on the personal computer in order to display the results in the three different formats discussed in section 2.4. We paid attention to the adequacy of the measured signals, the mechanics of the processes, and the adjustments that could be made during measurements subsequently. We overlooked the possible contamination of data due to aliasing .

The key information to be obtained from the processed data are the resonant frequencies and the $Q$ of each resonance. Both are given 
graphically in the plot in the $s$ plane. A number of false frequencies are also displayed, but the printed plots and CRT displays show clearly the correct ones, indicated by an exact repeat of the pole for the four different runs of Prony's algorithm. The exact numbers are then obtained from tabulated data. Bar plot plots of the magnitude of the residues offer additional help in identifying false high frequency poles, which are indicated either by a very small residue or for larger residues by lack of repetition for the four runs. The values of $Q$ are given in another bar plot. The $Q$ plots can be used by EMC engineers to determine an approximate enhancement of electromagnetic fields by the resonances.

\subsection{Sample Measured Data}

Three sets of sample data are given. The first is a simulation based on a generated waveform using known frequencies, damping $f a c t o r s$ and amplitudes. These results are helpful in learning how to interpret the plots. The second set of data is from the Apache helicopter. The third set of data is from the Black Hawk helicopter. There may be contamination in both of the real sets of data; the relatively few clear resonances identified should be verified by additional, independent tests.

Data from the measurements on the other aircraft are summarized in tables and figures.

\subsection{Simulated Data}

Figure 11 shows a plot of a computer-generated, time-domain waveform which contains 35 poles with frequencies of $7,17, \ldots 97,101,111, \ldots 191$, $214,234, \ldots 494$ megahertz. This set gives an example of contamination caused by Nyquist folding for frequencies above 250 megahertz. All the poles have complex residues with an amplitudes of 0.08. Although the number of poles is known in this case, we follow the procedure from section 2.4 and request a larger number of poles than 35. Four computer runs were made requesting 99, 98, 97 and 96 poles. The repetition of the location of the true poles is shown quite clearly, while the randomly generated poles do not repeat and hence do not overlay. The digitized waveform has 256 points with a sample spacing of 2 nanoseconds. The Nyquist folding frequency for this spacing is 250 megahertz. All the poles above 250 megahertz fold down and contaminate the data below 250 megahertz. The pole at 294 megahertz is 44 megahertz above 250 megahertz; it folds down to 206 megahertz. The pole at 494 megahertz is 244 megahertz above 250 megahertz; it folds down to 6 megahertz. Figure 12 is an expanded view of figure 11 . Figure 11 goes to 250 megahertz while figure 12 only goes to 60 megahertz. The residues of the folded-down poles are not changed by the folding; this is shown in figure 13. A bar plot of $Q s$ is shown in figure 14. The computer-calculated values of frequency are usually correct to about four significant figures. The computer-calculated values of the residues repeat to about the thirc significant figure for true poles, but vary much morie for the created poles. Table 1 is a computer printout of the numbers obtained from these runs. 


\subsection{Apache Data}

Figure 15 shows the s-plane plot of poles to 256 megahertz, while figure 16 is an expanded piot to 80 megahertz. The folding frequency for the sampling interval of 1.953 nanoseconds is 256 megahertz. Resonances appear at approximately 19, 38, 59 and 76 megahertz. The residue plot in figure 17 also confirms these as real poles. Figure 18 shows $Q$ values about $60,200,60$ and 70 , respectively. The printout of numbers in Table 2 shows the exact frequencies to be $18.6,38.3,59.1$ and 77.4 megahertz. Whether all these poles are real or are allased is not known since the raw data were not properly filtered before it was digitized, but if there is any doubt whether the Apache helicopters might be expected to operate in locations where external sources generate these frequencies, additional investigation is prudent.

\subsection{Black Hawk Data}

Figure 19 shows the s-plane plot of poles for the Black Hawk helicopter for frequencies to 256 megahertz. Figure 20 is the expanded plot for poles to 60 megahertz, and shows possible poles at approximately $11,21,28,39$ and 55 megahertz. These poles are not quite as closely clustered as those of the Apache, but they do show up consistently in data taken with the helicopter at other azimuths. Figure 21 of the complex residues casts doubt as to whether these poles are real in that the amplitudes do not repeat as closely as those of the Apache. Figure 22 shows the Qs to be $10,25,23,50$ and 50 respectively. The exact frequencies from table 3 are 10.9, 21.3, $28.3,38.2$ and 53.6 megahertz. These data show some scatter and hence may not be real. The 21.3 and 28.3 megahertz frequencies should be examined by additional measurements, since both are in frequency bands authorized by the FCC for amateur radio service, and in addition, the 21.3 megahertz frequency is very close to the 21.4 megahertz intermediate frequency which may be used in various avionic systems.

\subsection{Data From Other Helicopters}

The Chinook we used for measurements had one rotor blade removed for service, so the data were not taken on a complete aircraft.

One figure of the expanded pole plot is given for each aircraft in figures 23 through 27. Tables 4 through 8 give data of the first 50 poles, residues, and $Q s$ for each of the aircraft following each figure. The same caveat applies to all of these data that applied to the earlier data - some valid information is present, but before it is used in any critical application, the measurements should be repeated with better analog filtering to eliminate any possible contamination due to aliasing.

The processed data from the other helicopters and one fixed-wing aircraft are summarized in table 9 to identify the resonant frequencies. The data for the $0 H 58$ were taken with a larger time interval between samples, giving a lower folding frequency (128 megahertz), and may be more contaminated by aliasing than the data for the other aircraft. Specifically, resonances are indicated at 3 and 5 megzhertz in figure 27, lower than is possible based on the maximum dimension of this aircraft. 


\subsection{Summary and Recommendations}

A new measurement system was used to determine the natural resonant frequencies of helicopters by radiating the aircraft with short pulses, receiving the reflected waveforms, and by using the mathematical transform known as Prony's algorithm to extract the natural resonant frequencies of these helicopters. Many improvements have already been made in all parts of the system; more are anticipated. One procedural error was made in our measurements. The analog data were not adequately filtered before digitization. This casts suspicion on the data taken, but the measurement procedure and system have been successfully demonstrated at frequencies and for applications not possible previously.

Three additional recommendations are made for any subsequent work. Adequate filtering should be applied. Full-horn antennas should be used to measure horizontal E field polarization, since the major dimension of a helicopter is in the horizontal plane. The effect of the helicopter image below the ground screen should be evaluated using scale models. This should be done for both antenna polarizations.

\subsection{Acknowledgments}

This work was supported by the US Army Aviation Systems Command for both the development of the measurement system and arrangements for the testing at Ft. Rucker. Gilmore Krener of AVSCOM made special arrangements for these tests at the US Army Aviation Development Test Activity at Ft. Rucker. L. Eagerton, M. White and C. Mello provided excellent support at Ft. Rucker.

Roland A. Sweet of the Applied Math Division developed the software to implement Prony's Algorithm and Linda Lindgren wrote the computer code.

\subsection{References}

[1] Poggio, Andrew J., et al, "Evaluation of a Processing Technique for Transient Data," IEEE Trans. Ant and Prop., Vol AP-26, No. 1, Jan. 1978.

[2] Van Blaricum, M.L., and Mittra, R., "Problems and Solutions Associated with Prony's Method for Processing Transient Data," IEEE Trans. Ant. and Prop., Vol. AP-26, No. 1, Jan. 1978.

[3] Van Blaricum, M.L., and Mittra, R., "A Technique for Extracting the Poles and Residues of a System Directly from Its Transient Response," IEEE Trans. Ant. and Prop., Vol. AP-23, No. 1, Nov. 1975.

[4] Baum, C.E., "On the singularity expansion method for the solution of electromagnetic interaction problems," Interaction Note 88, Dec. 11, 1971.

[5] Tesche, Frederick M., "On the Analysis of Scattering and Antenna Problems Using the Singularity Expansion Technique," IEEE Trans. Antennas ani Propagat., vol AP-21, pp 53-62, Jan. 1973. 
[6] Beneking, H., and Filensky, W., "The GaAs MESFET as a Pulse Regenerator in the Gigabit per Second Range," IEEE MTT Vol. MTT-24, Jun. 1976.

[7] Liechti, C.A., Special Issue on Microwave Field-Effect Transistors, IEEE Trans. Microwave Theory and Techniques, Vol. MTT -24, Jun. 1976.

[8] Lawton, R.A., and Ondrejka, A.R., "Antennas and the Associated Time Domain Range for the Measurement of Impulsive Fields," NBS Tech Note $1008,1978$. 


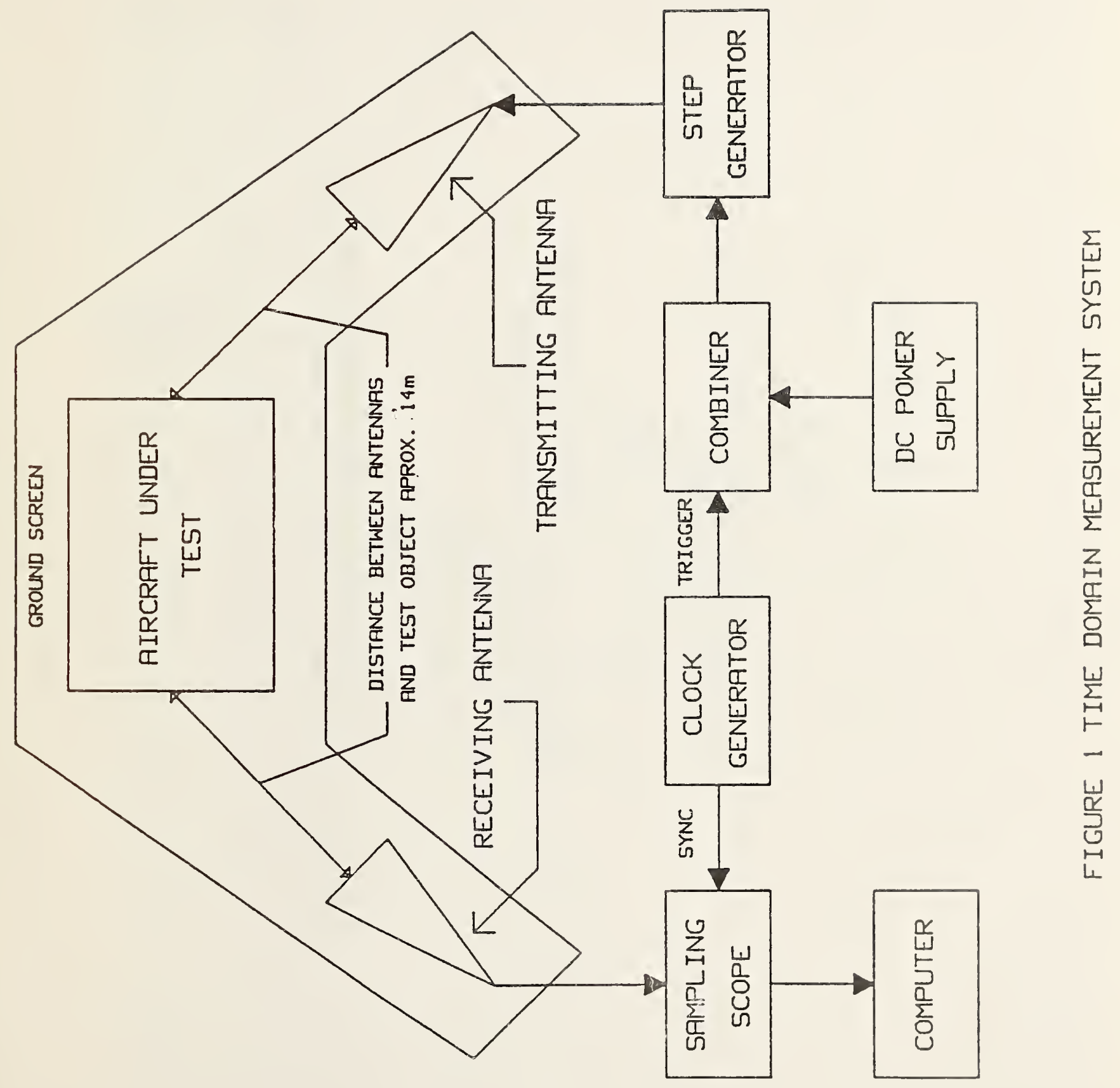




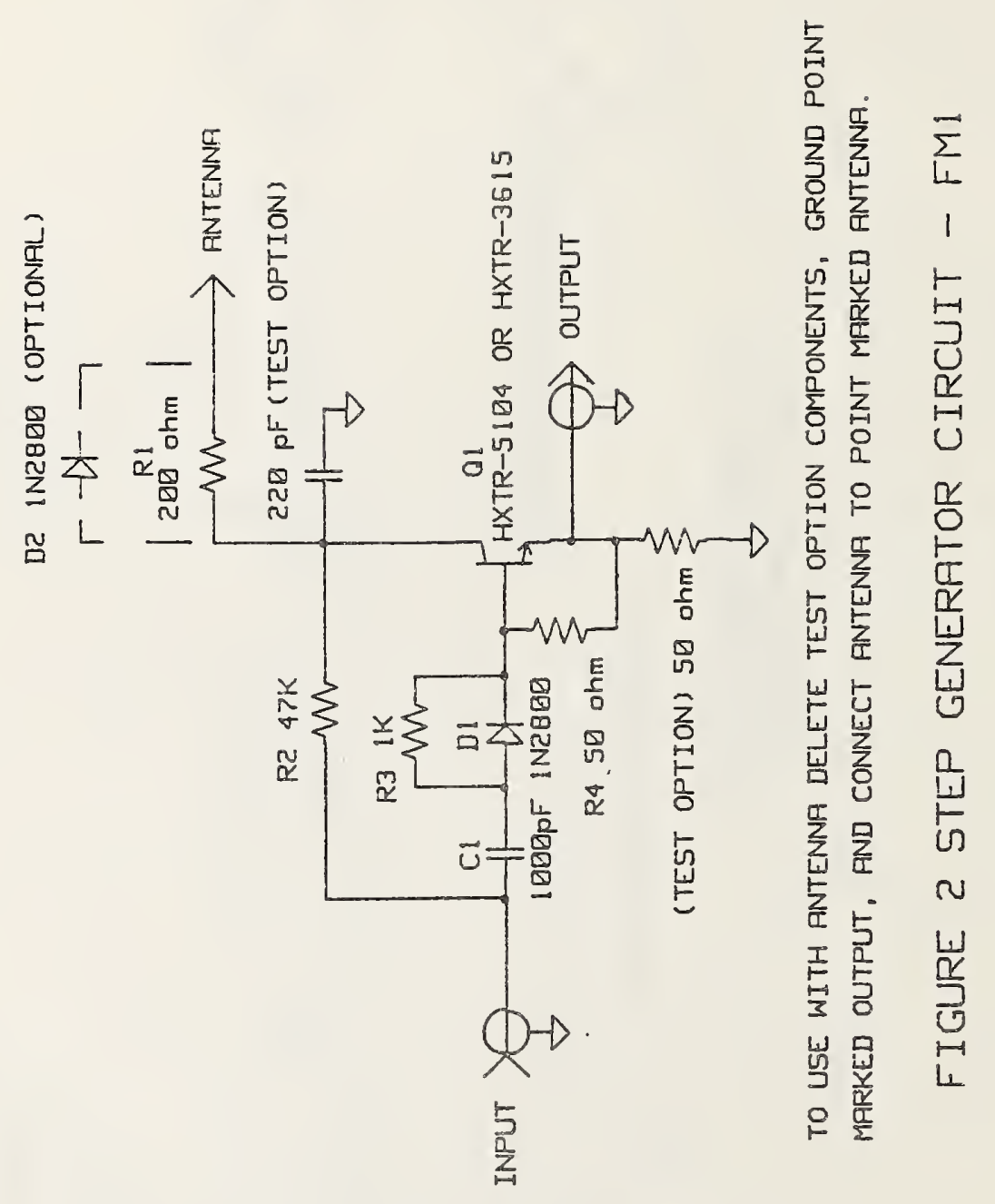




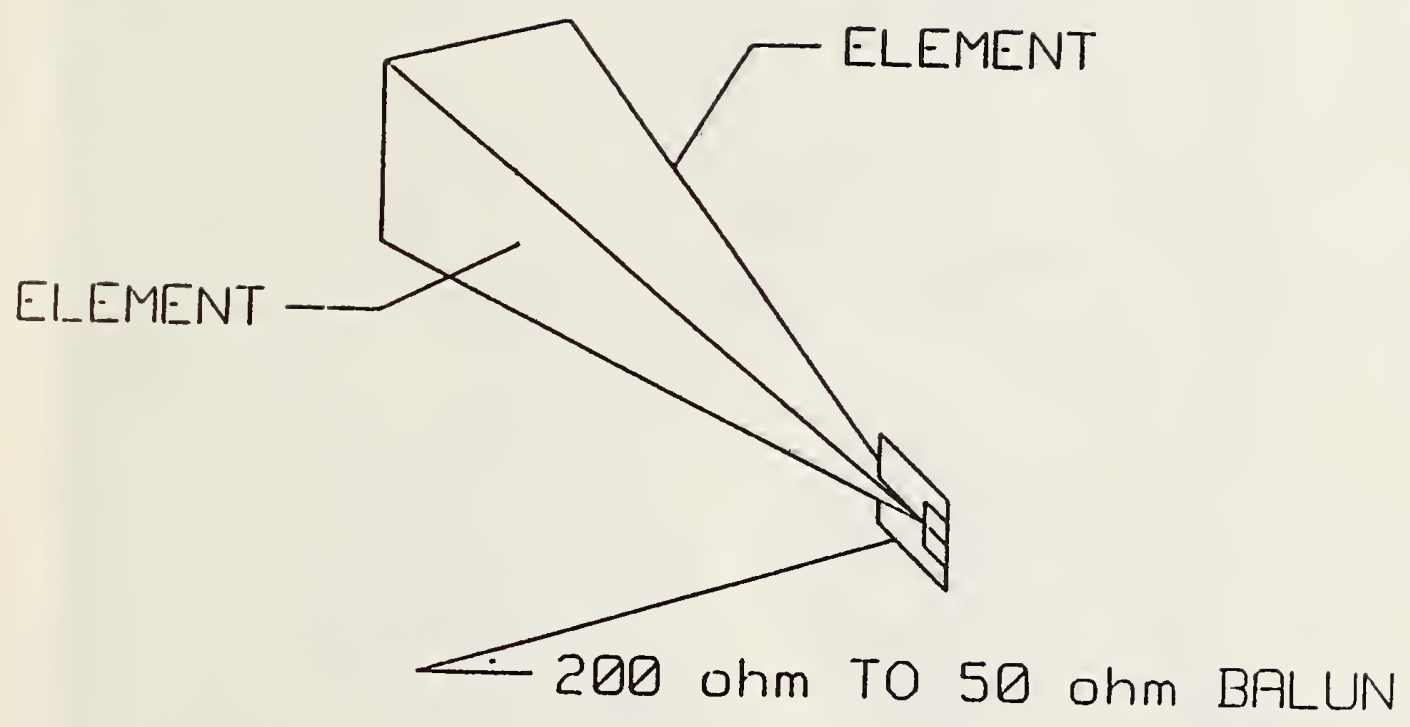

Figure 3. Balanced TEM horn antenna drawings. 


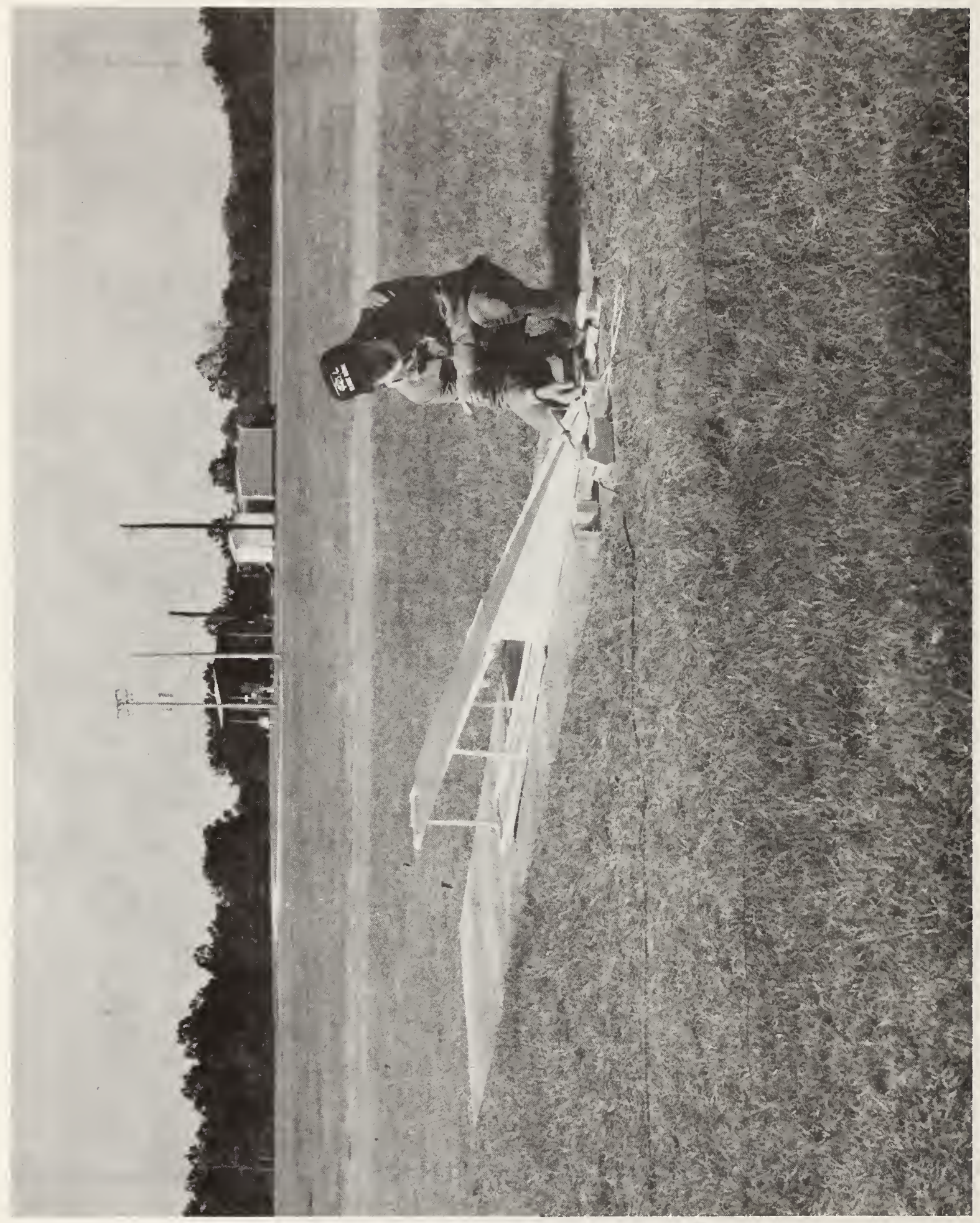


$403 \mathrm{mV} / \mathrm{div} 20 \mathrm{~ns} / \mathrm{div}$

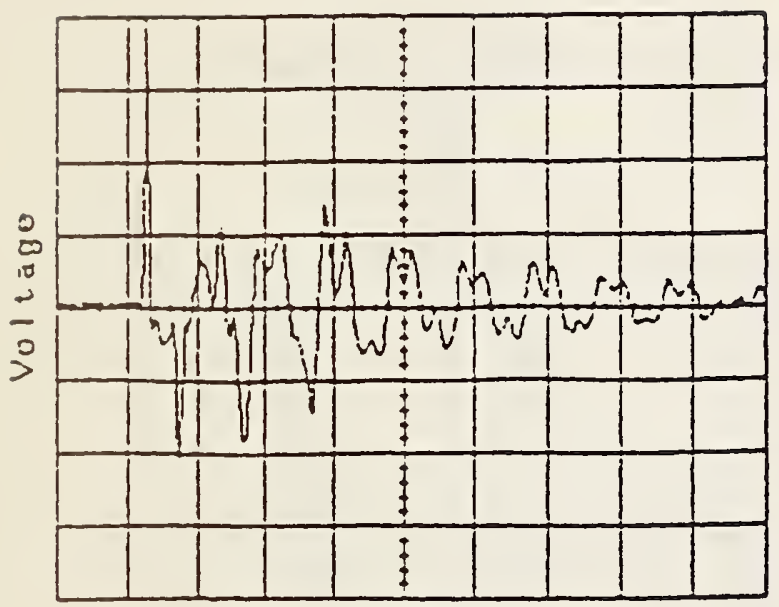

Time

Low Impedance Mismatch

$154 \mathrm{mV} / \mathrm{div} 20 \mathrm{~ns} / \mathrm{div}$

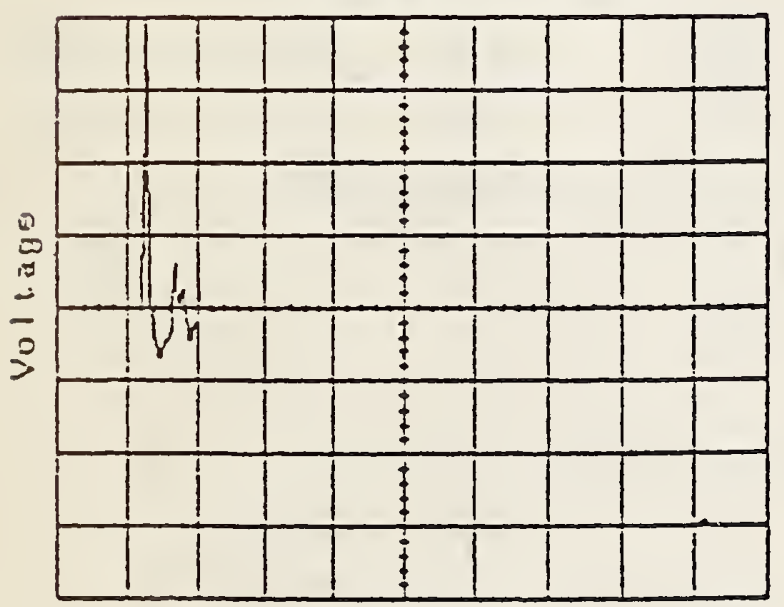

Time

200 Ohm Matched
$82.1 \mathrm{mV} / \mathrm{div} 20 \mathrm{~ns} / \mathrm{div}$

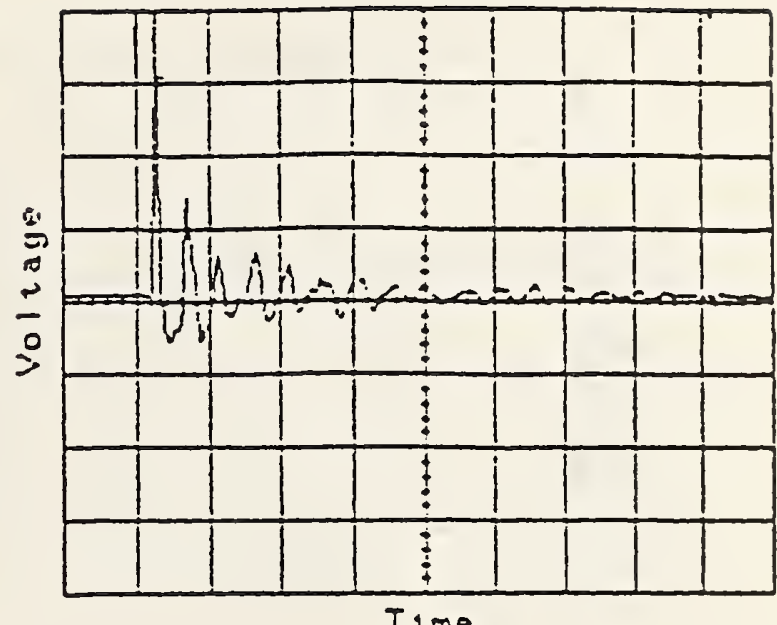

High Impedance Mismatch

$359 \mathrm{mV} / 0 \mathrm{iv} 20 \mathrm{~ns} / 0 \mathrm{iv}$

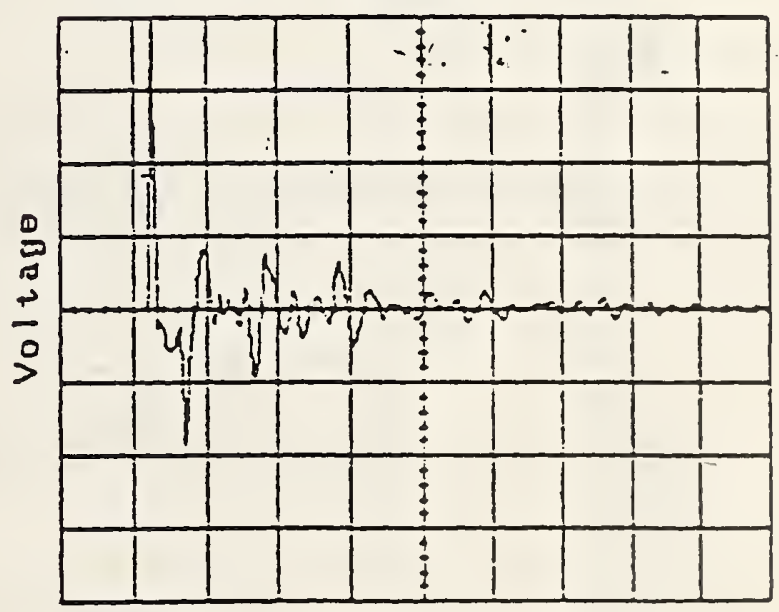

TIme

Modified 200 Onm Matched (Diode Across R1)

Figure 5. Signal flots shoving the difference beiveen matched and unmatched anienna response. 


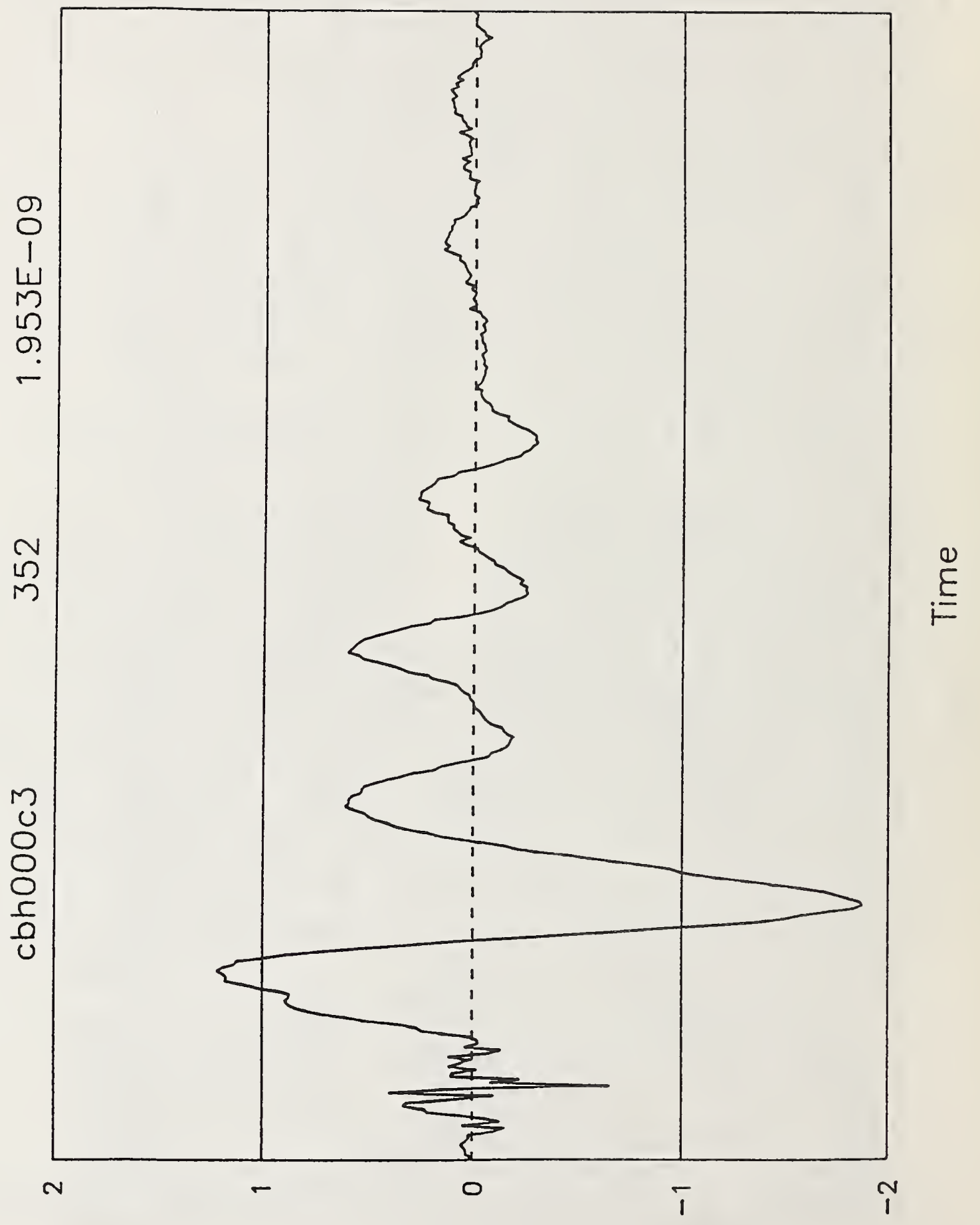

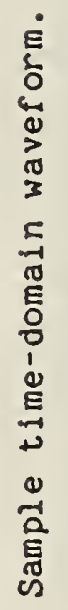


$\begin{array}{llll}0 & 0 & 0 & 0 \\ \sigma & \infty & \hat{\sigma} & 0 \\ \sigma & \sigma & \sigma & \\ \times & \triangleright & \# & 0\end{array}$

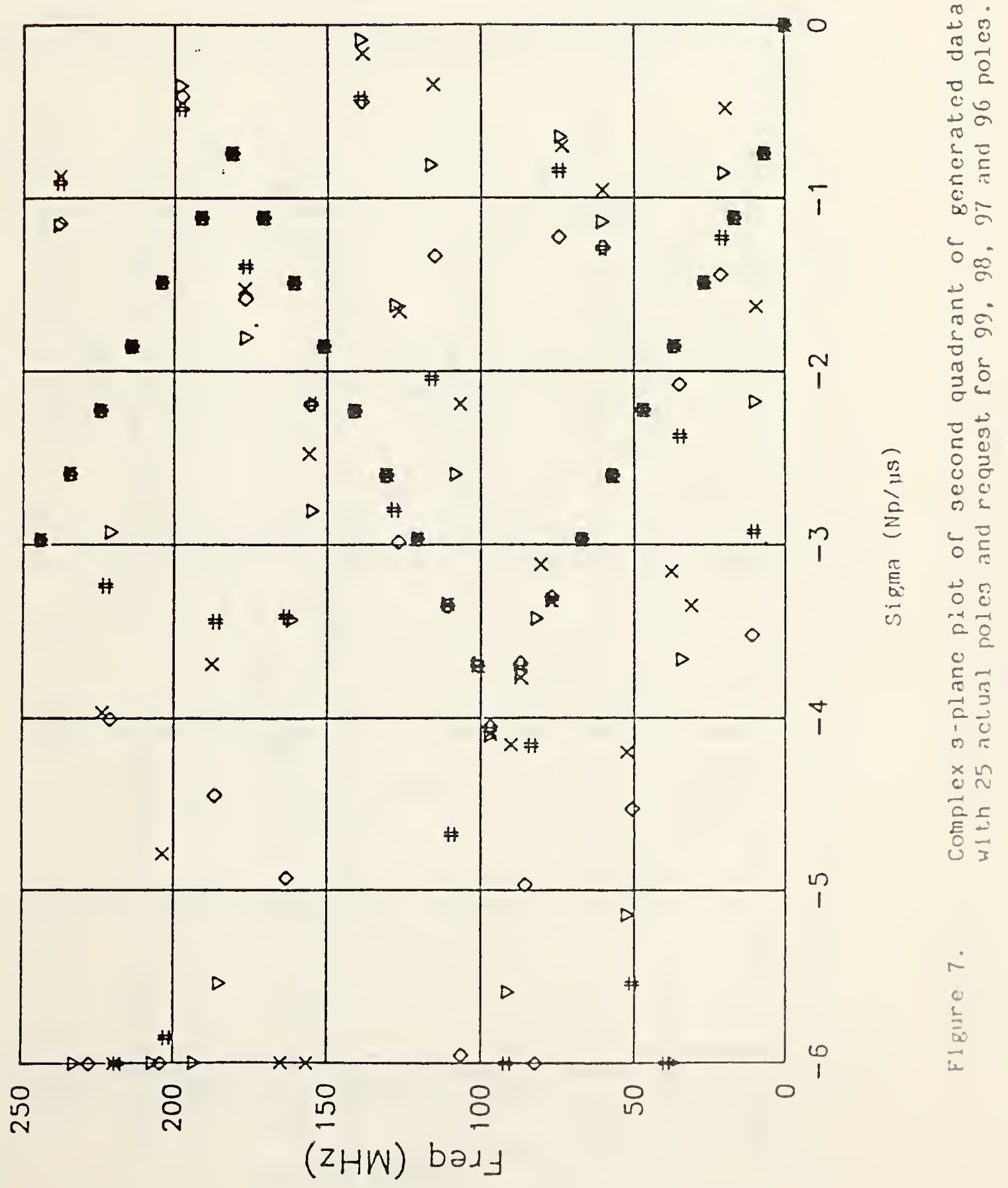




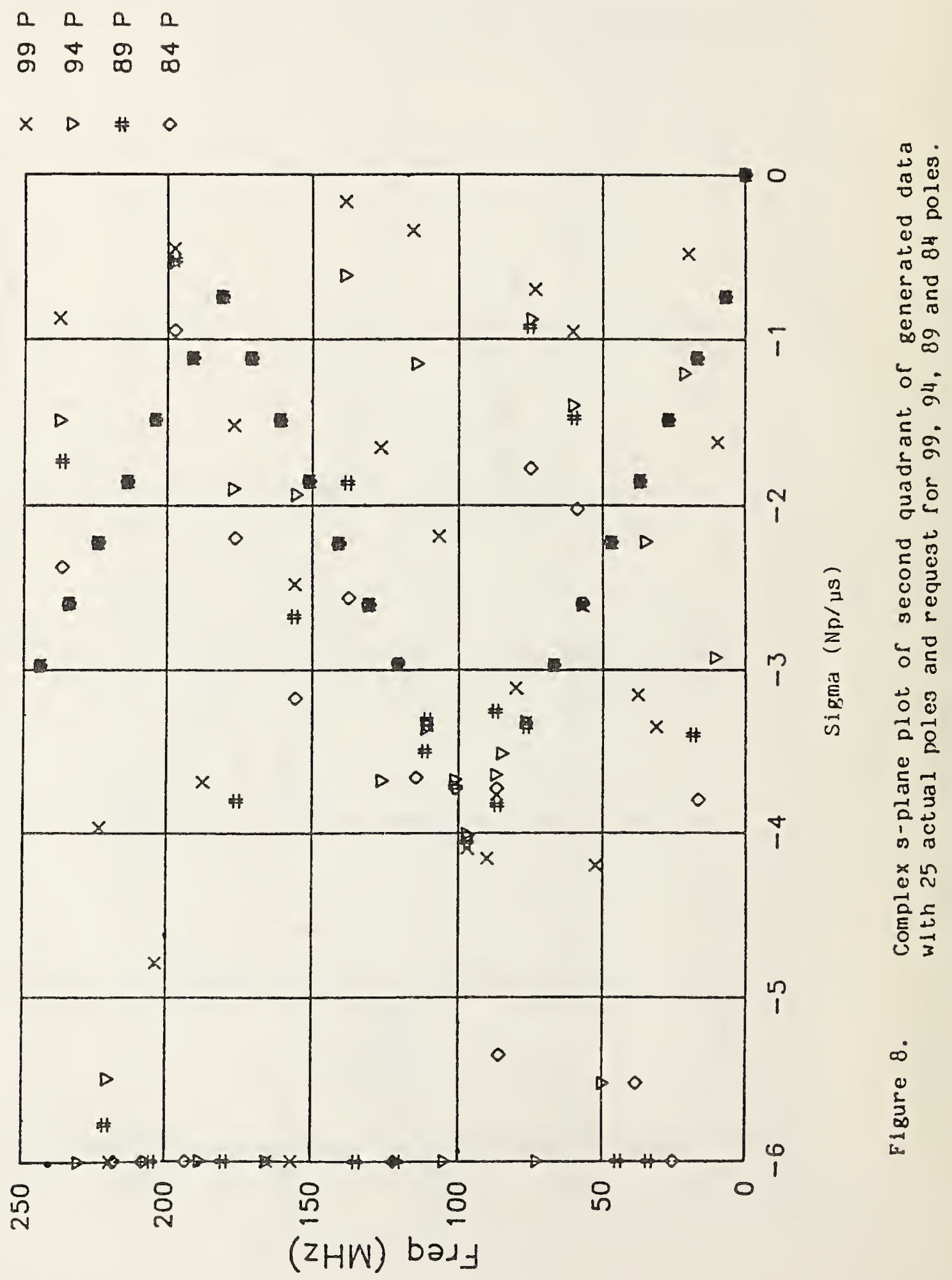




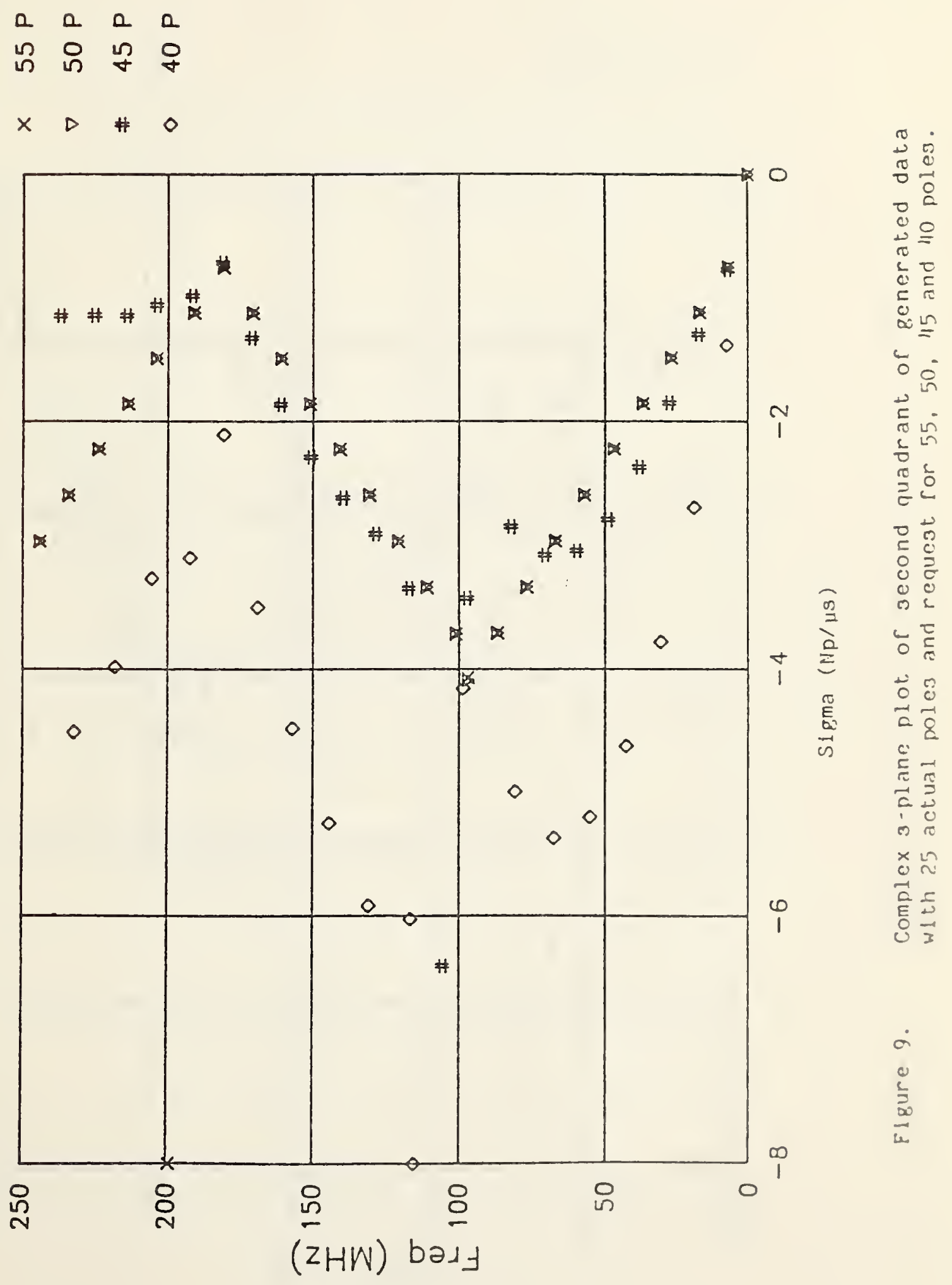




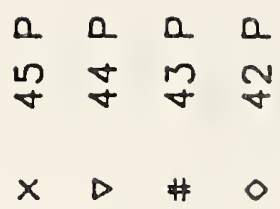

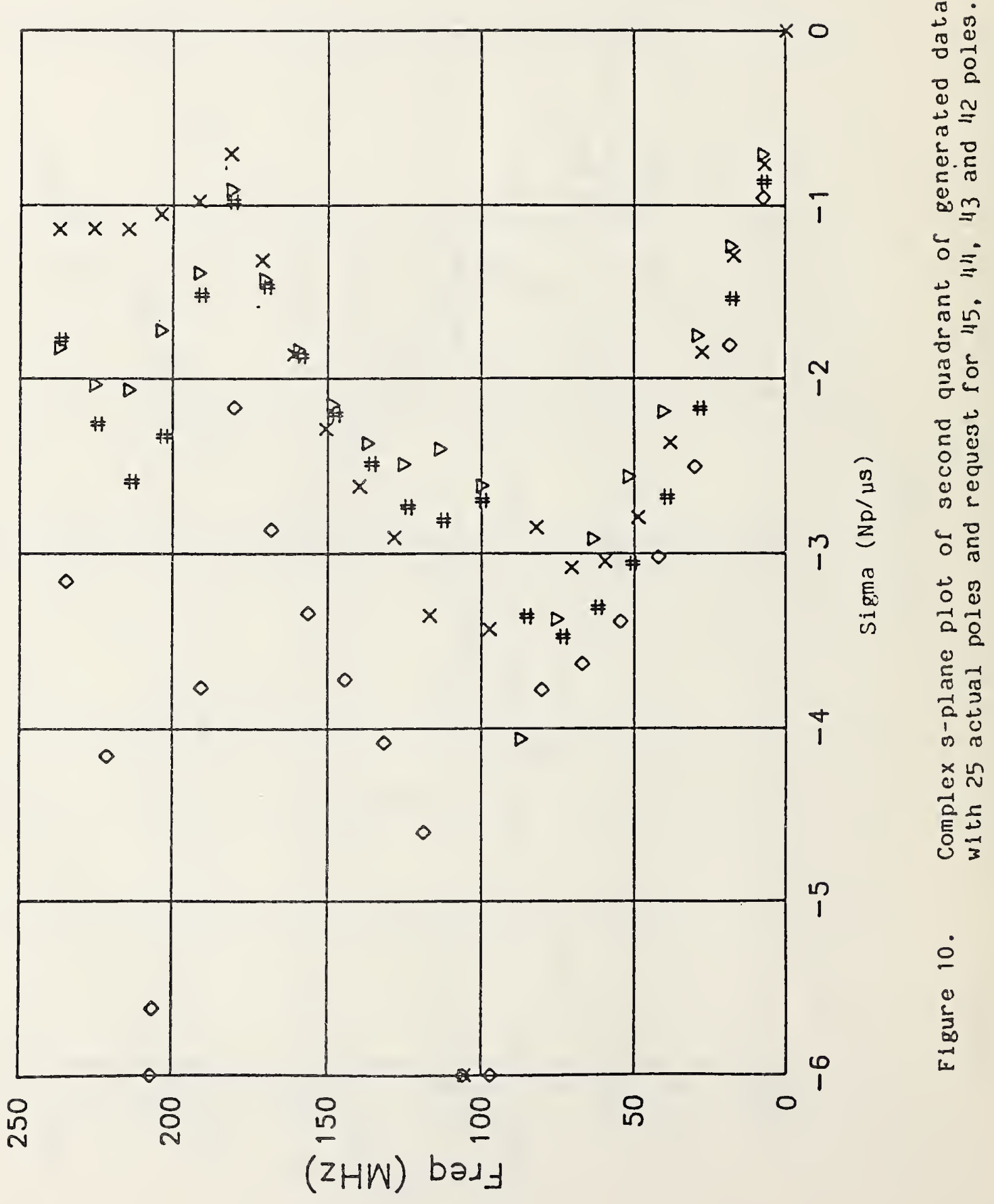



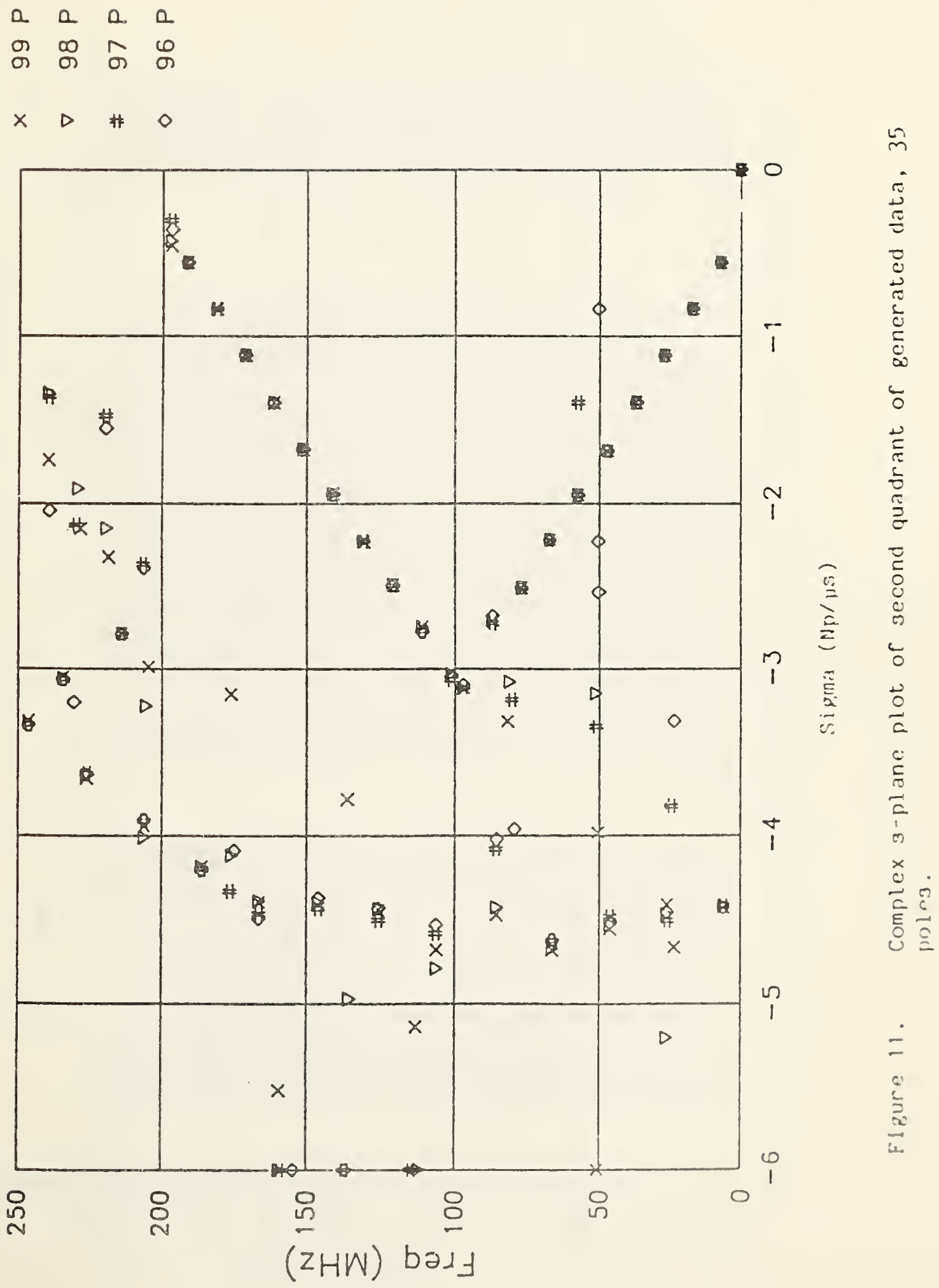
$\begin{array}{llll}0 & 0 & 0 & 0 \\ \sigma & \infty & \hat{\sigma} & 0 \\ \sigma & \sigma & \sigma & \sigma \\ \times & 0 & \# & 0\end{array}$

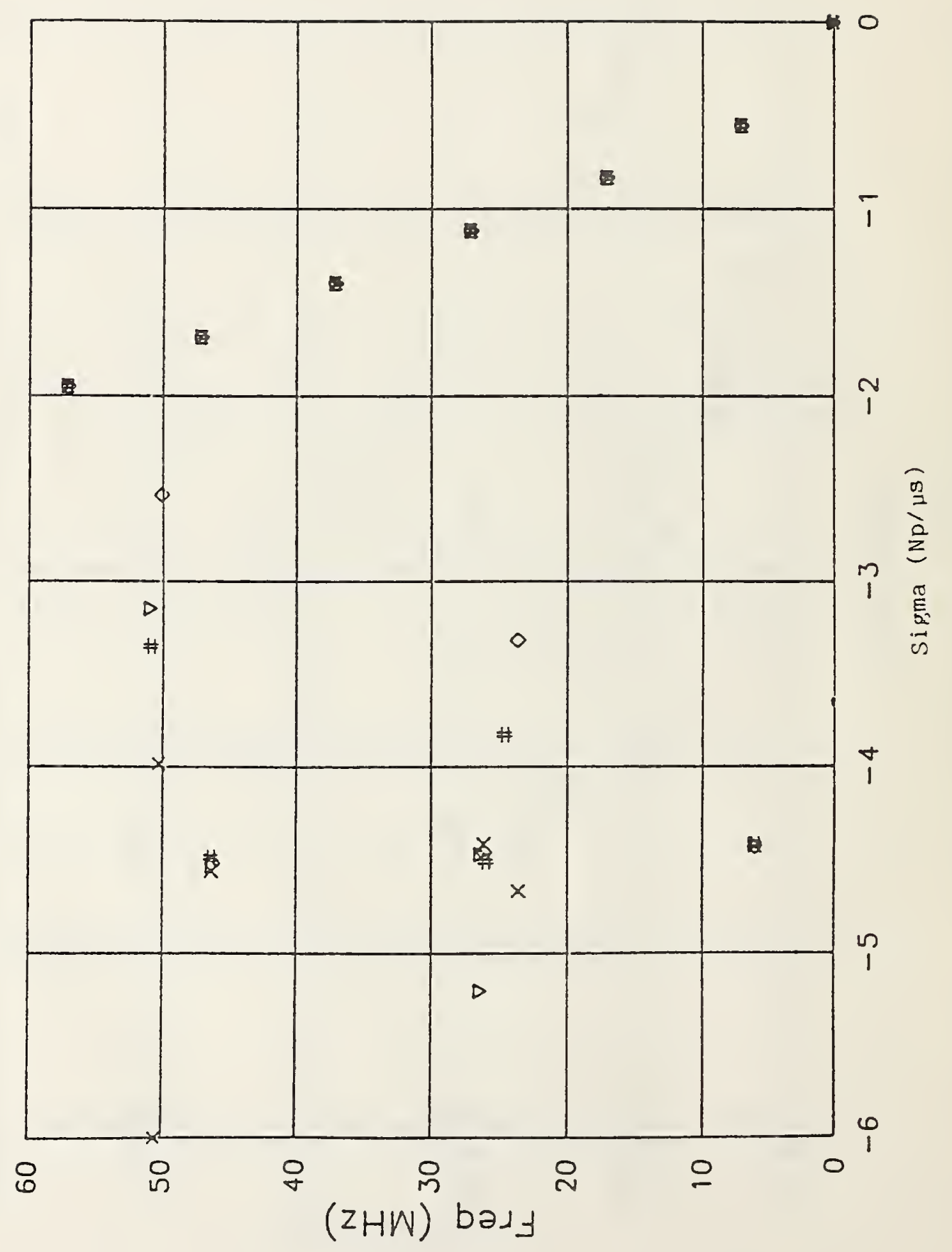

0
0
0
0
0
0
0
0
0
0
0
0
0
0
0
0
0
0
0
0
0
0
0
0
0
0
0
0
0
0
0
0
0
0
0
0
0
0
0
0
0
0
0
0
0
0
0
0
0
0

$i$
0
$\vdots$
$i=1$ 


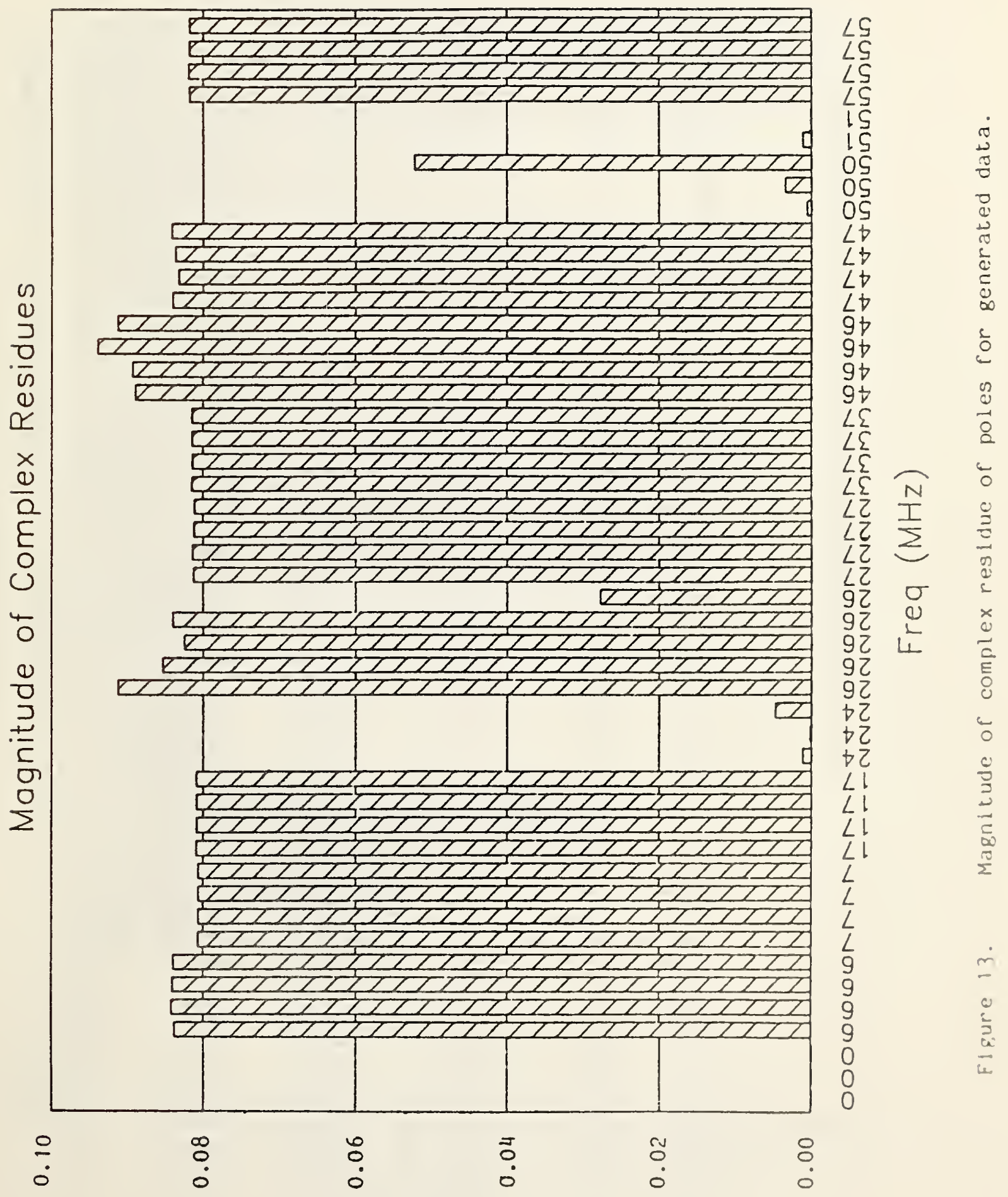




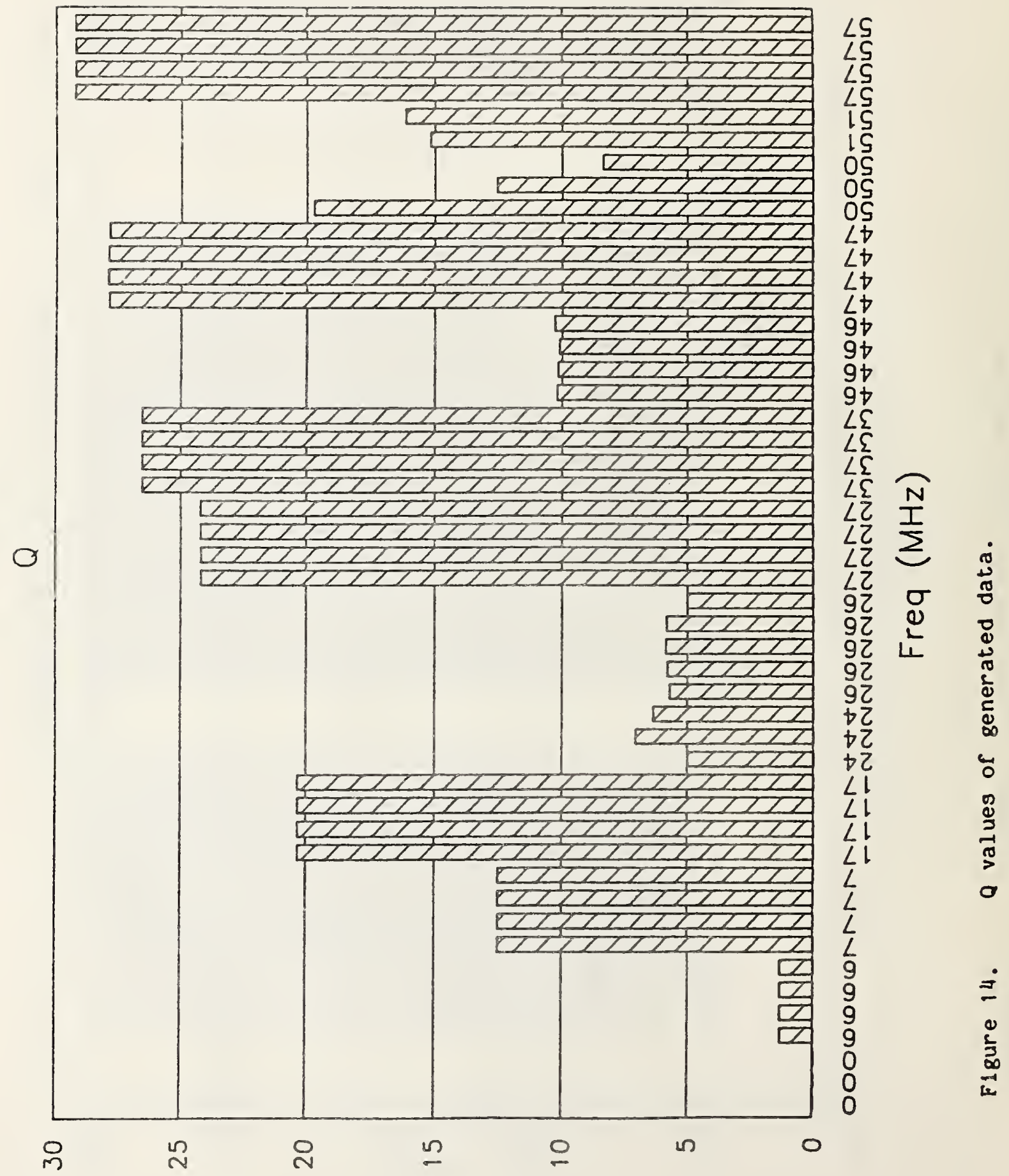


Table 1. Data from main-frame computer of simulated signal. Table gives processed data of real and imaginary parts of complex frequency, real and imaginary parts of complex residue, $Q$, magnitude of residue, sigma and frequency.

$\begin{array}{ccccc}\begin{array}{c}\text { Complex } \\ \text { Freouency }\end{array} & \text { Resioue } & \text { Q Mag } & \text { Sigma Freq } \\ \text { Real Imag } & \text { Real } & \text { Imag. } & \text { Res } & (\mathrm{Np} / \mu \mathrm{S}) \text { (MHz) }\end{array}$

\begin{tabular}{|c|c|c|c|c|c|c|c|}
\hline & & & & & & & \\
\hline & & & & 1 & $0.45-2$ & -4.4 & \\
\hline 429900 & $9866 e 6$ & $-1.90 e-2$ & -.040515 & 1 & $8.4 e-2$ & -4.4 & 6.0 \\
\hline - & $47 \in 6$ & $-1.07 €-2$ & .040513 & i & E. $4 e-2$ & -4.4 & 6.0 \\
\hline 4499 & & $-1.9<e-2$ & .040357 & 9 & 8. $4=-2$ & -4.4 & 5.0 \\
\hline-557 & & $3.207 e-3$ & $-.040 \div 9$ & 13 & $9 e-2$ & -.6 & 7.0 \\
\hline-53 & & $3.272 e-3$ & -.04017 & 13 & $9 e-2$ & -.6 & 7.0 \\
\hline-55 & & $3.275 e-3$ & $-.040 i 7$ & 93 & 8.1e-2 & -.6 & 7.0 \\
\hline$-5 \xi$ & 1 & $3.260 e-3$ & -.040173 & 13 & $6.1 e-2$ & -.6 & \\
\hline & & $5.103 e-3$ & $.040 i i 3$ & 20 & E. ie-2 & -.8 & 97.0 \\
\hline & 1 & $5 . i 23 e-3$ & $-.0 \div 00$ & 20 & 8. $1 \in-2$ & -.8 & 17.0 \\
\hline & 1 & Le -3 & -.0400 & 20 & 8. $1 e-2$ & -.8 & . \\
\hline & & $1 e-3$ & -.0401 & 20 & E. 1e-2 & -.8 & 17.0 \\
\hline $466:$ & $2.359 e 7$ & $-4.49 e-4$ & .0004083 & 5 & $1.2 e-3$ & -4.7 & 23. \\
\hline & 2.35 & $1.079 e-4$ & -.0000 & 7 & $2.6 e-4$ & $-3 \cdot 3$ & $\cdot$ \\
\hline & 2 & $2.160 e-5$ & -.0021 & 6 & $4.8 e-3$ & -38 & \\
\hline $1=$ & \&2e 7 & $-7.75 e-3$ & .0425 & 6 & $9.9 e-2$ & -4.5 & $2 \vdots$. \\
\hline 45 & $2.5009 e 7$ & $-7.58 e-3$ & .0419 & 6 & $8.5 e-2$ & -4.5 & 26.0 \\
\hline 4 & $2.6027 \varepsilon$ & $-7 . i 1 \in-3$ & .04053 & 6 & 8. $2 e-2$ & -4.4 & 26.0 \\
\hline 45 & 6247 & 6. $4 ;$ He -3 & $.01: 9$ & 5 & 8. Le-2 & -4.5 & 26.2 \\
\hline & $\epsilon=8$ & $-9.36 e-2$ & .0015 & 5 & $2.6 e-2$ & -5.2 & 26. \\
\hline 191 & 2.6 & $6.932 e-3$ & .04002 & 24 & $8.9 e-2$ & -1.1 & 27.0 \\
\hline 13 & 2. Egoge 7 & $7.052 e-3$ & -.010069 & 24 & $8.9 \in-2$ & $-i . i$ & 27.0 \\
\hline 1 & 2.6009 & $6.93 i e-3$ & -.039986 & 24 & $8.1 e-2$ & -1.9 & 27. \\
\hline 1 & & 6.63 & -.039 & 24 & $8.1 e-2$ & $-1 . i$ & 27. \\
\hline & & $8.6^{\circ}$ & -.039774 & 27 & $8.1 e-2$ & -9.4 & 37.0 \\
\hline$-i 392$ & & $8.723 \mathrm{e}$ & -.039731 & 27 & $6.9 e-2$ & -9.4 & 3 \\
\hline 13927 & 3.7 & $8.703 e-3$ & .039754 & 27 & $8.1 e-2$ & -9.4 & \\
\hline 2 & 3. & $8.697 e-3$ & -.039 & 27 & 8. $1 e-2$ & -1.4 & 37 \\
\hline$=$ & 4.5922 & -4.7 & .044 & 10 & 8. $0 e^{-2}$ & -4.5 & 46. \\
\hline $15=$ & 4.6935 & $-3.70 e-3$ & $.04 \div 593$ & 10 & 6. $g \in-2$ & -4.5 & \\
\hline ームララ & 4.5169 & $-5.47 e-3$ & .046555 & 10 & $9.4 e-2$ & -4.6 & 4 \\
\hline$-246 i 9$ & $4.5202 e 7$ & $-3.23 e-3$ & .045 & 90 & Q. $1 e-2$ & -4.5 & 46. \\
\hline 6 & $4 . t 09$ & $1.90 i e-2$ & .0404 & 28 & 8. $4 e-2$ & -9.7 & 47. \\
\hline & 4.599 & $1.10 z e-2$ & -.0400 & 28 & $8.3 e-2$ & -9.7 & 47. \\
\hline & 4.699 & $1.108 e-2$ & .0402 & 28 & $8.4 e-2$ & -9.7 & 47. \\
\hline$-i 68 \equiv 2$ & 4.6999 & $1.087 e-2$ & -.040 & 28 & $8.4 e-2$ & -1.7 & 47.0 \\
\hline & $5.002^{\circ}$ & $9.700 e^{-4}$ & -.0002 & 20 & 6. He-4 & -2.5 & 50. \\
\hline & 5.02 & $-7.05 e-4$ & -.009 & 13 & $3.5 e-3$ & -4.0 & 50. \\
\hline & 5.049 & $2.607 \mathrm{e}-2$ & $-.00 i i$ & 8 & $5.2 €-2$ & -6.0 & 50. \\
\hline$-32 !$ & $5.0857 \mathrm{e}^{\circ}$ & $-5.03 \epsilon-4$ & -.000344 & 15 & 1. $2 e-3$ & -3.3 & 50. \\
\hline & 5.0905 & $-8.06 e-5$ & -.00003 & 16 & $1.7 e^{-4}$ & -3.2 & 50. \\
\hline & & 9. $225=e-2$ & -.0350 & 29 & 8. $2 e-2$ & -9.9 & 57. \\
\hline & & $1.225 e-2$ & -.0390 & 29 & 8. $2 e-2$ & -1.9 & 57. \\
\hline & $=0$ & $1.295 e-2$ & -.03? & 29 & 8. $2 e-2$ & -9.9 & 57. \\
\hline & & $9.230 e-2$ & $-.0 \Xi E \Xi 5$ & 29 & $\varepsilon . \hat{z} \in-2$ & -9.0 & 57. \\
\hline
\end{tabular}


$\begin{array}{llll}1 & 0 & 0 & 0 \\ \sigma & \infty & \hat{\sigma} & 0 \\ \sigma & \sigma & 0 & 0\end{array}$

$\times \triangleright \# 0$

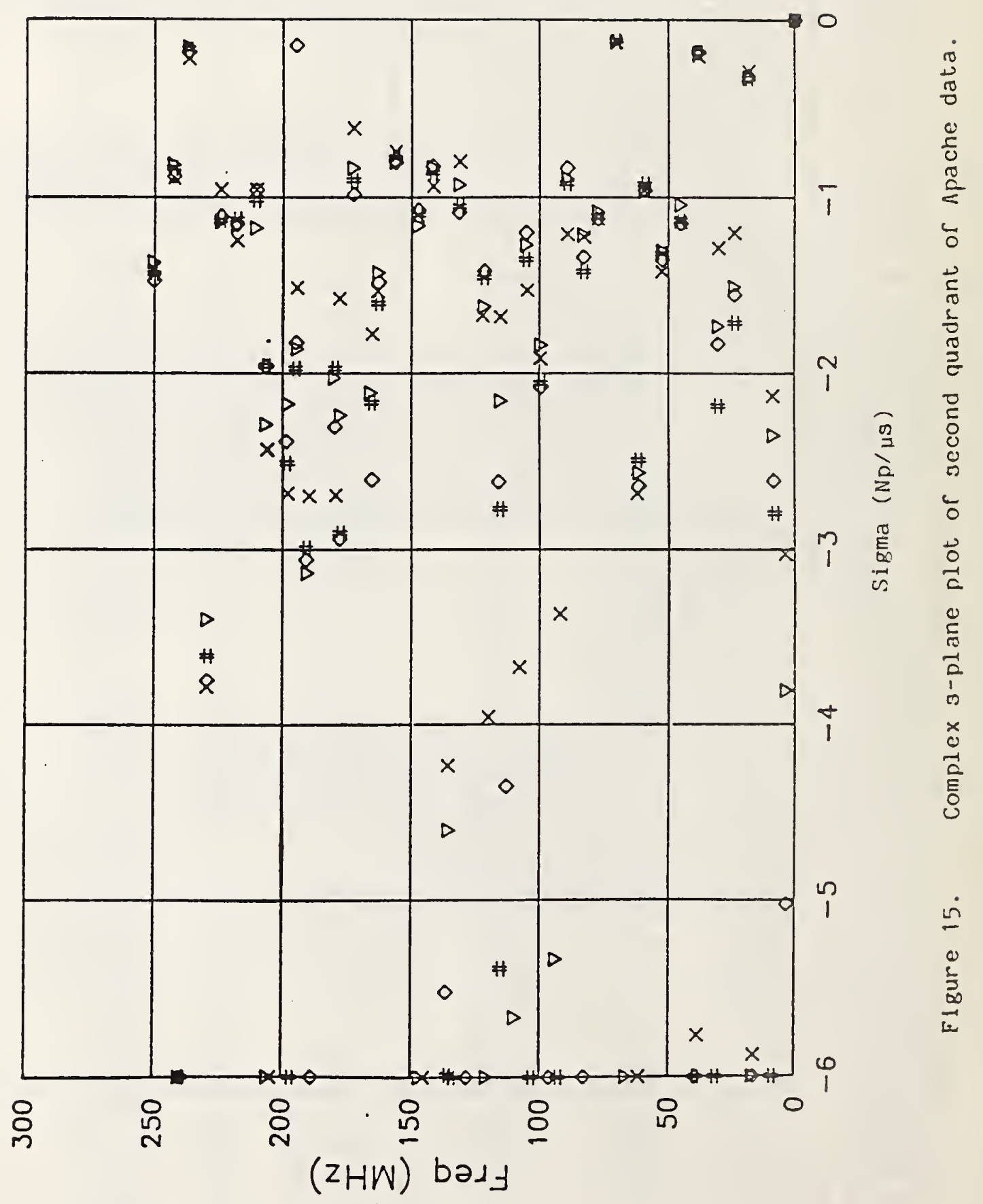


$\begin{array}{llll}0 & 0 & 0 & 0 \\ \sigma & \infty & \hat{n} & 0 \\ \sigma & 0 & 0 & 0 \\ \times & 0 & \# & 0\end{array}$

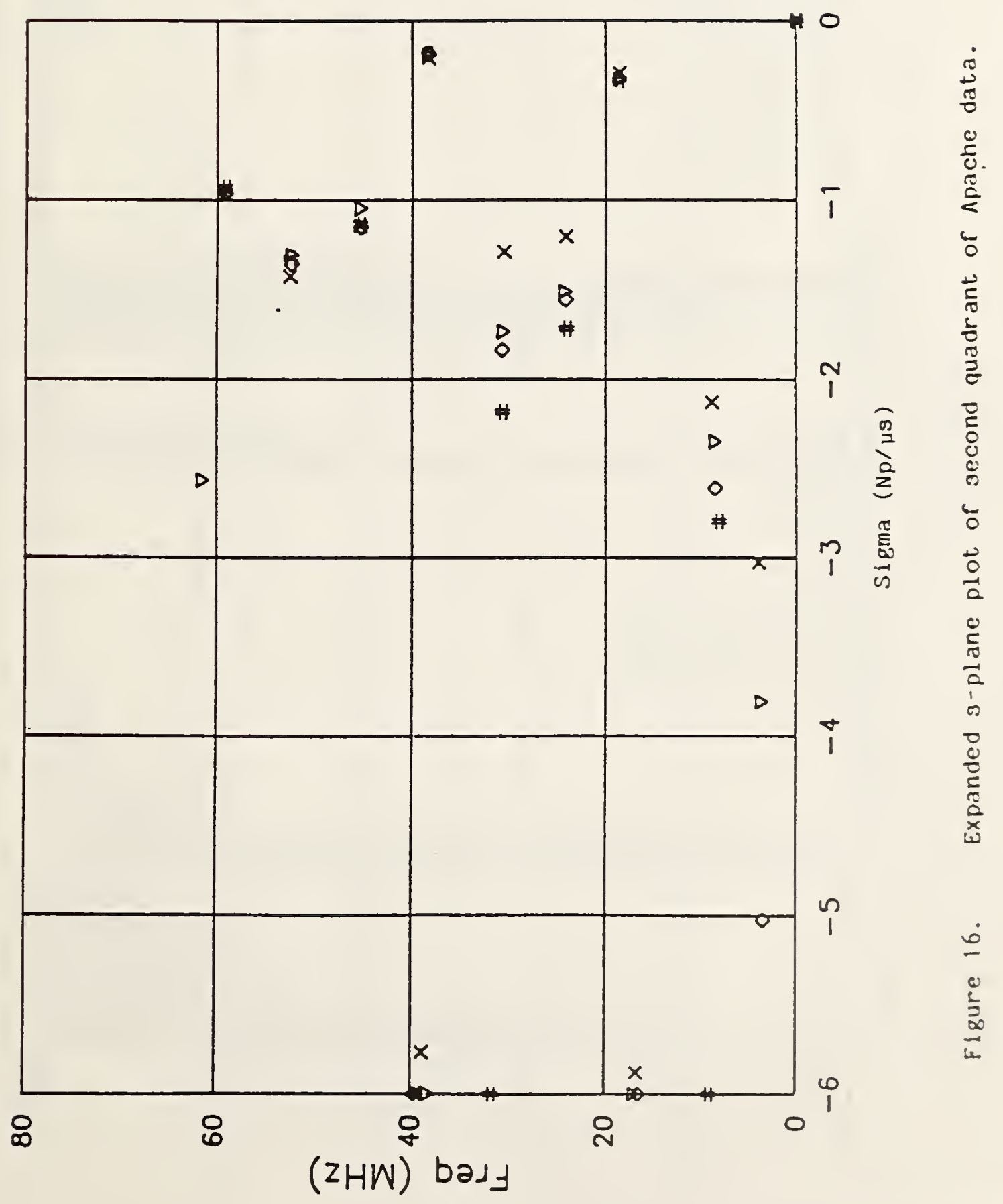




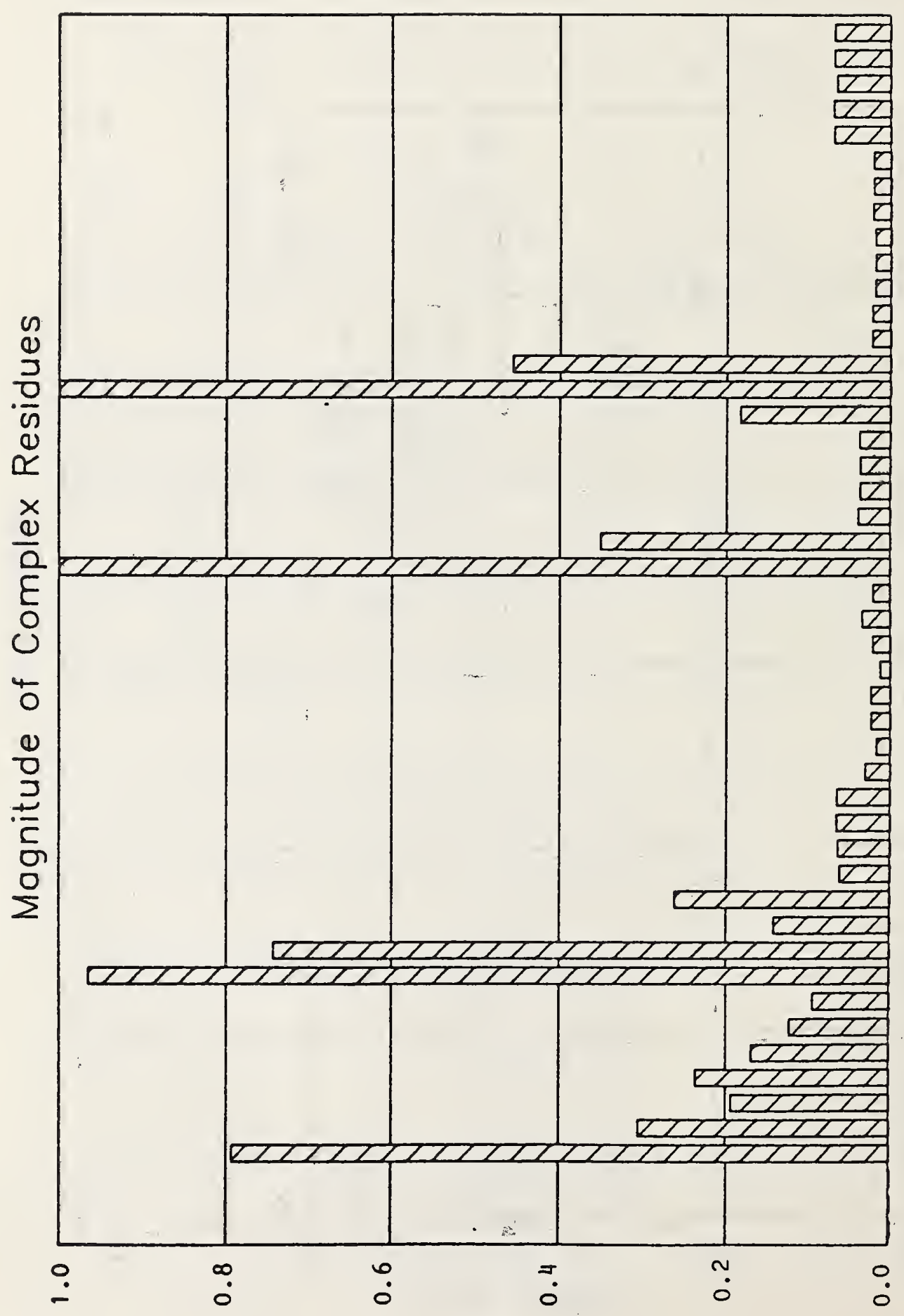

$\begin{array}{ll}19 & \\ 65 & \\ 65 & 5 \\ 6 S & 5 \\ 65 & 5\end{array}$

¿S

25

25

St

St

St

07 <क

$6 \varepsilon \quad \delta \frac{\pi}{\pi}$

6ह $8 \varepsilon$

8र $\quad-5$

$8 \Sigma \widehat{~} 8 \Sigma$ ஸे

彾 N

เह $\sum$ 更

เह

Oह $\sigma: 0$

记

㕸 L

$\rightarrow 2$

$\rightarrow 2$

6 !

$6 !$

61

61

$<1$

6

6

6

6

a)

ํㅡㅁ

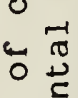

。

$3 \rightarrow$

考

एँ

$=$

产 


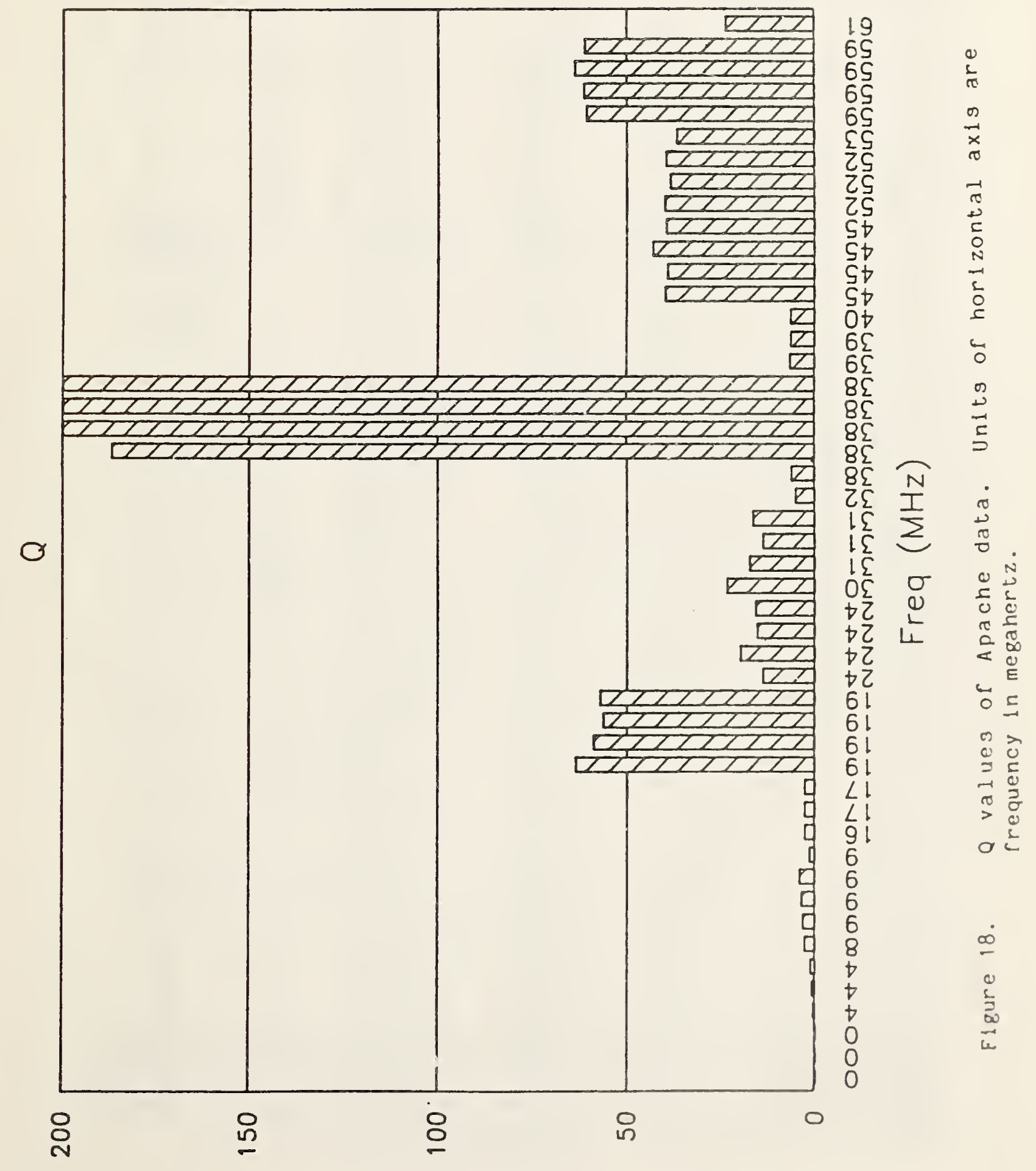


Table 2. Data from main-frame computer from Apache signal. Table gives processed data of real and imaginary parts of complex frequency, real and imaginary parts of complex residue, $Q$, magnitude of residue, sigma and frequency.

\begin{tabular}{|c|c|c|c|c|c|c|}
\hline \multirow{2}{*}{\multicolumn{2}{|c|}{$\begin{array}{l}\text { Complex } \\
\text { Ereouercy }\end{array}$}} & Fes: & & \multirow[t]{2}{*}{ Q } & \multirow{3}{*}{$\begin{array}{l}\text { Ma: } \\
\text { Re: }\end{array}$} & \multirow{3}{*}{$\begin{array}{cc}\text { Sigma } & \text { Freq } \\
(\mathrm{Np} / \mu \mathrm{S}) & (\mathrm{M} i \mathrm{z})\end{array}$} \\
\hline & & & & & & \\
\hline ñeEl & $I m \bar{c} \bar{E}$ & $\bar{F} \in \bar{E}$ & $I \Pi=g$ & & & \\
\hline
\end{tabular}

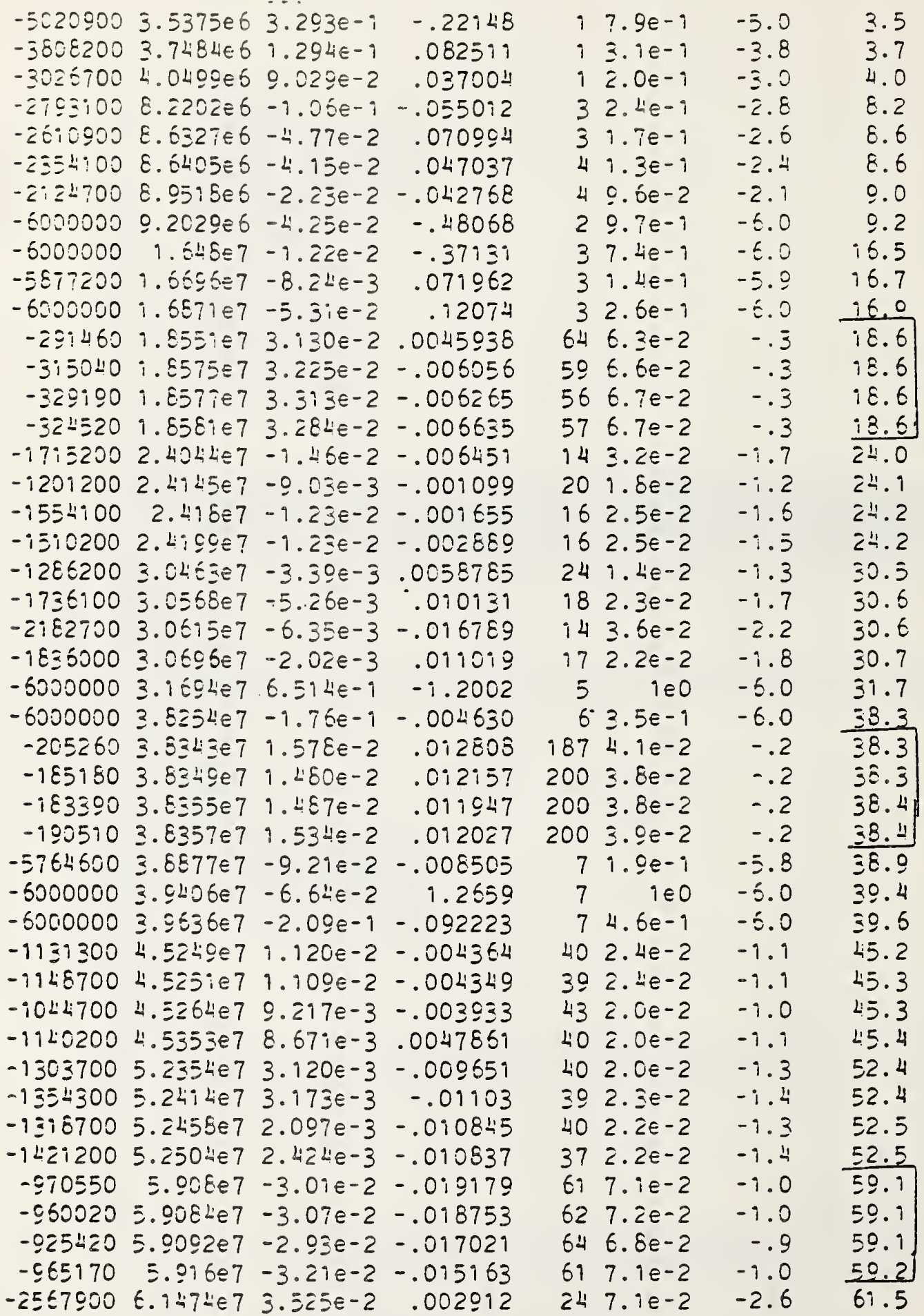




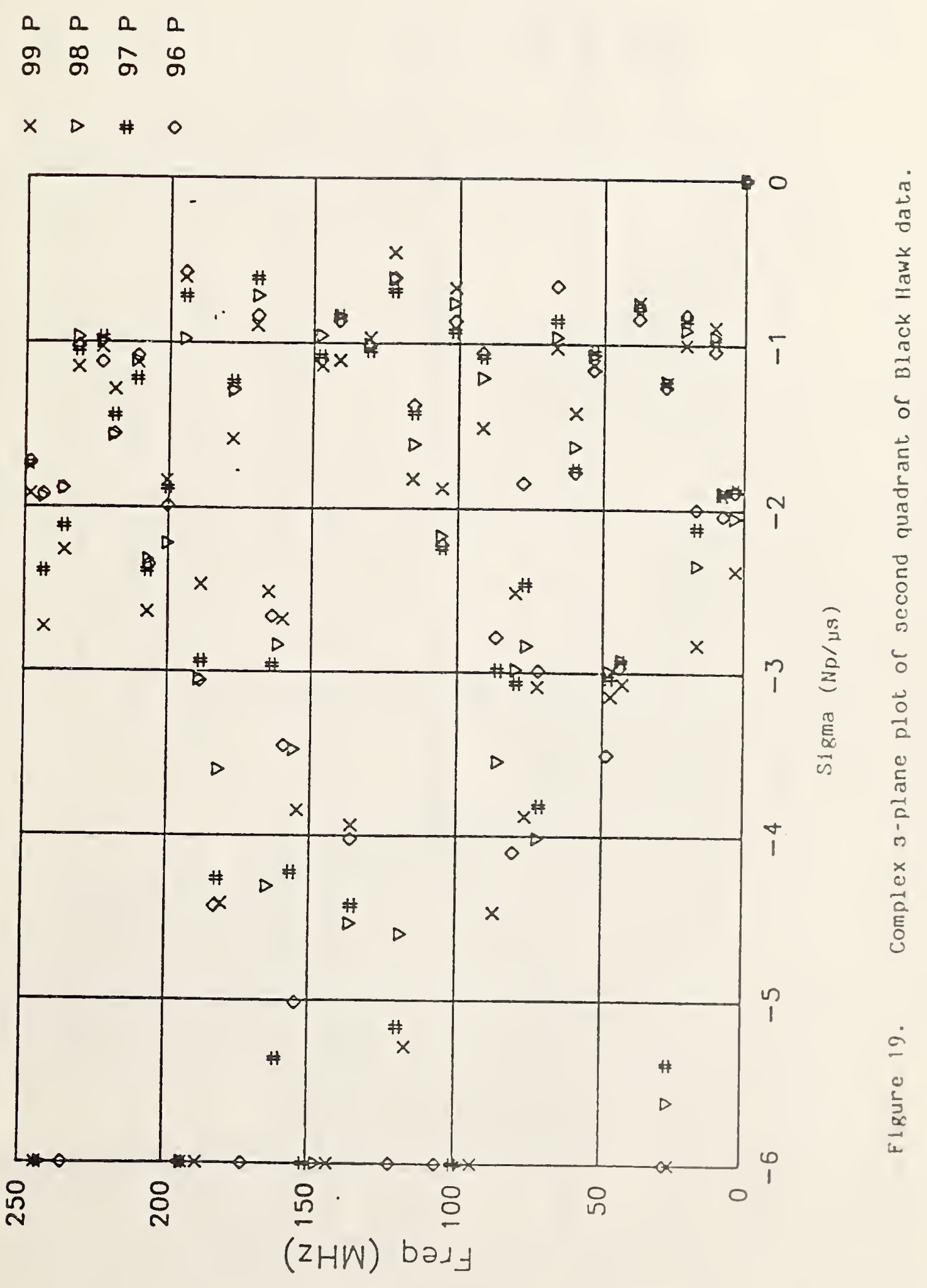



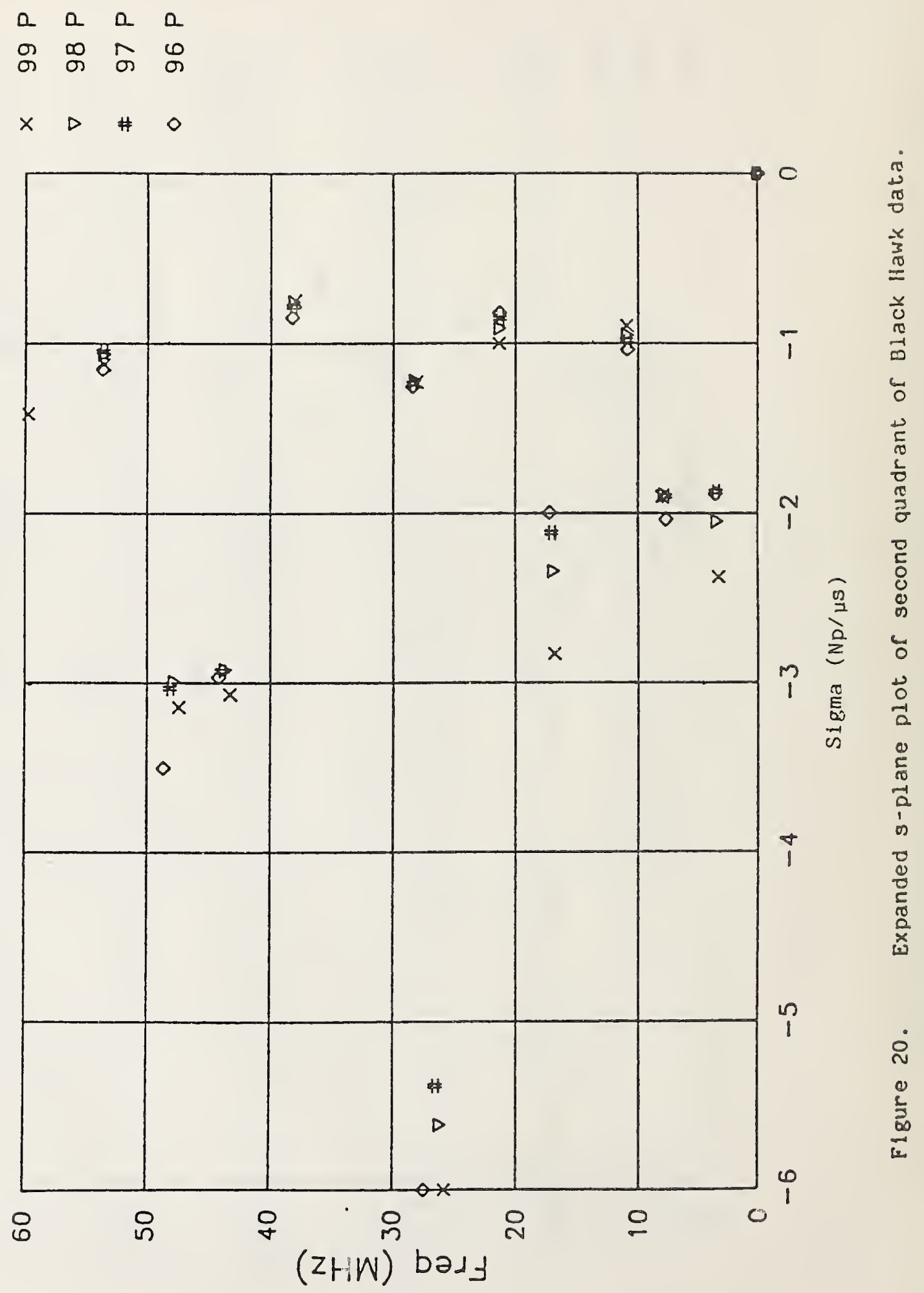


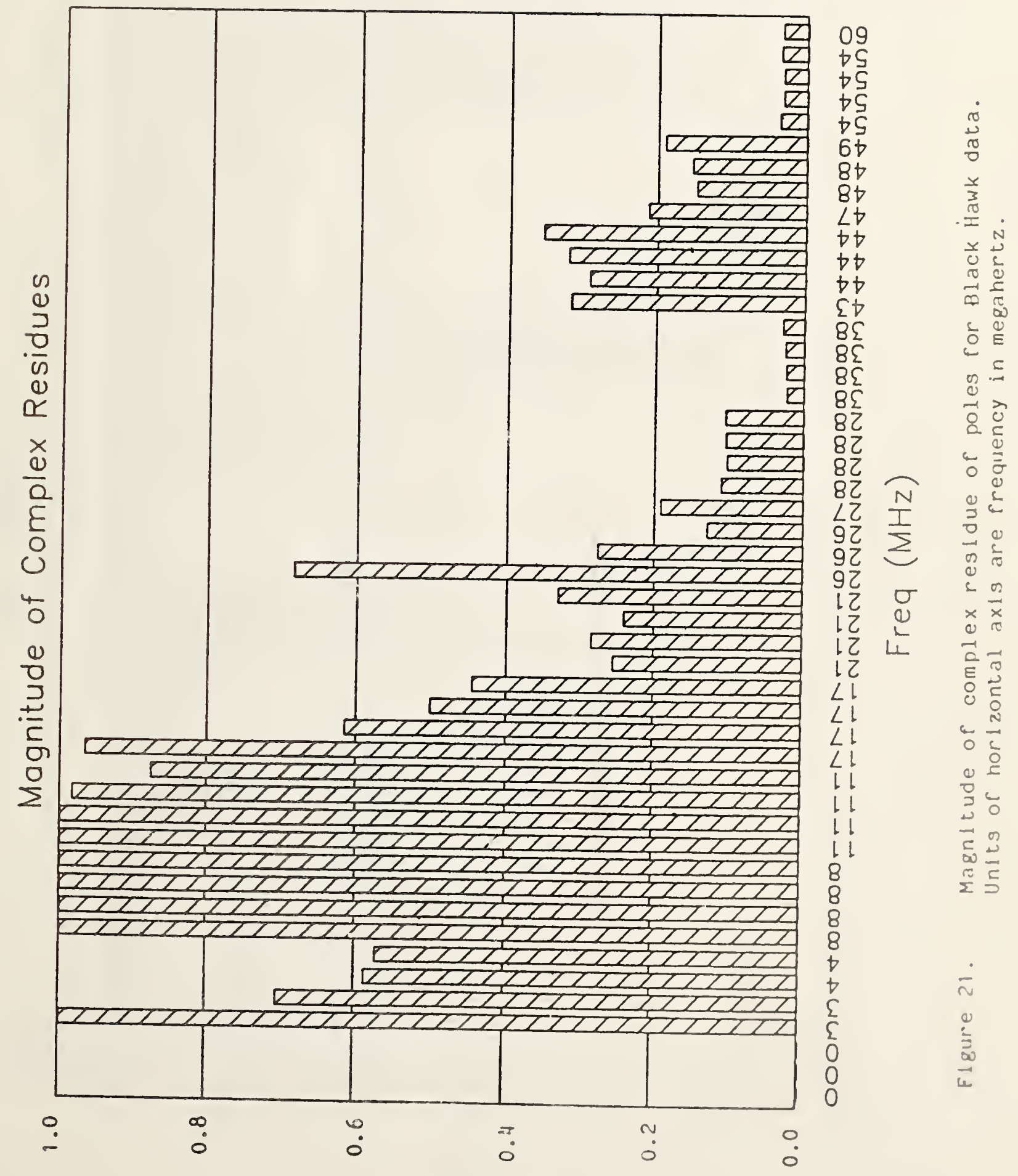




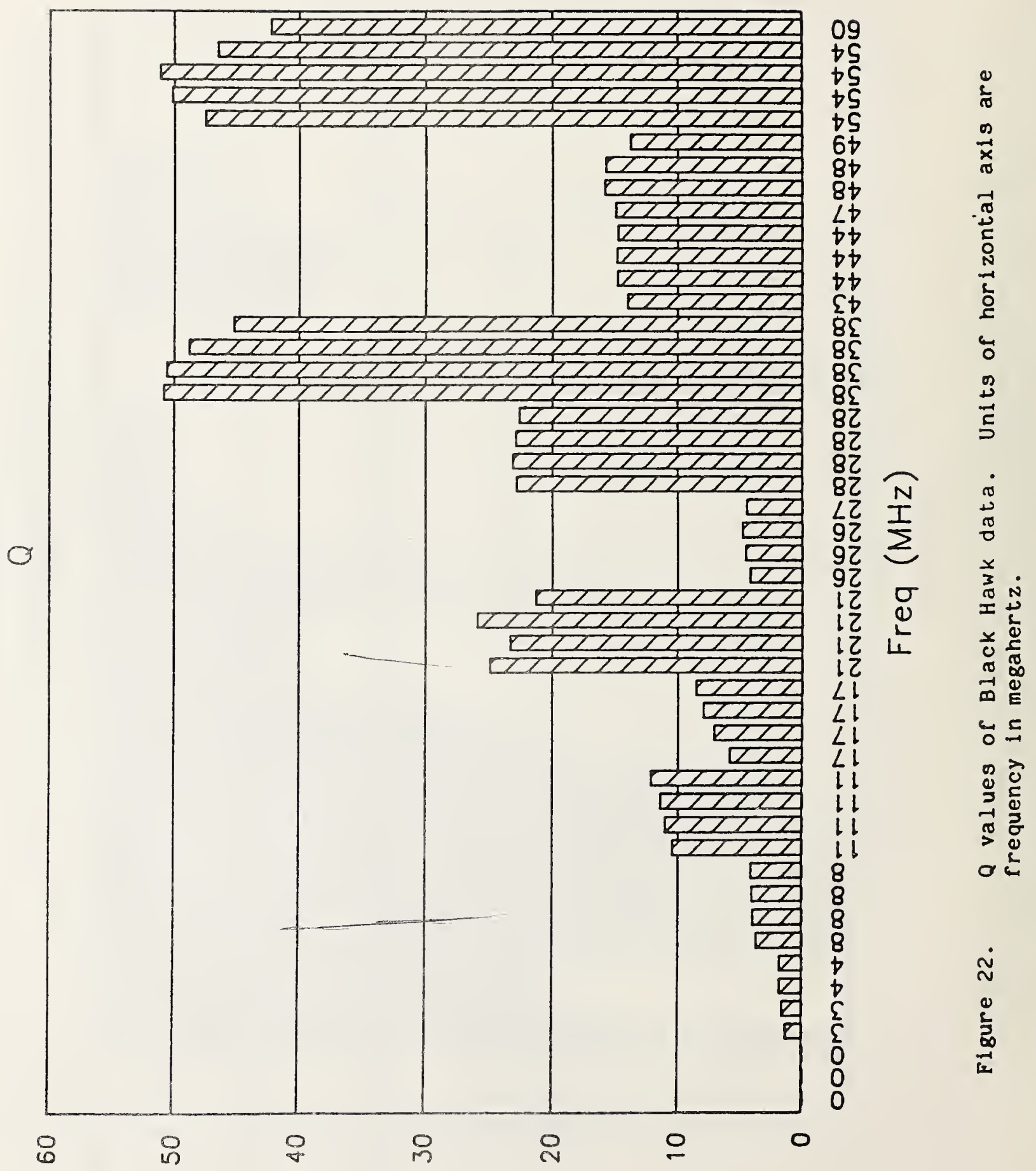


Table 3. Data from main-frame computer from Black Hawk signal. Table gives processed data of real and imaginary parts of complex frequency, real and imaginary parts of complex residue, Q, magnitude of residue, sigma and frequency.

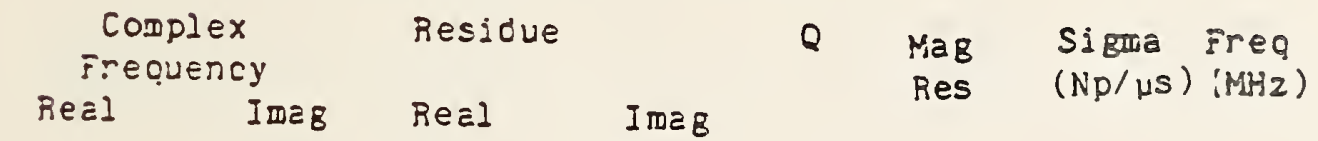

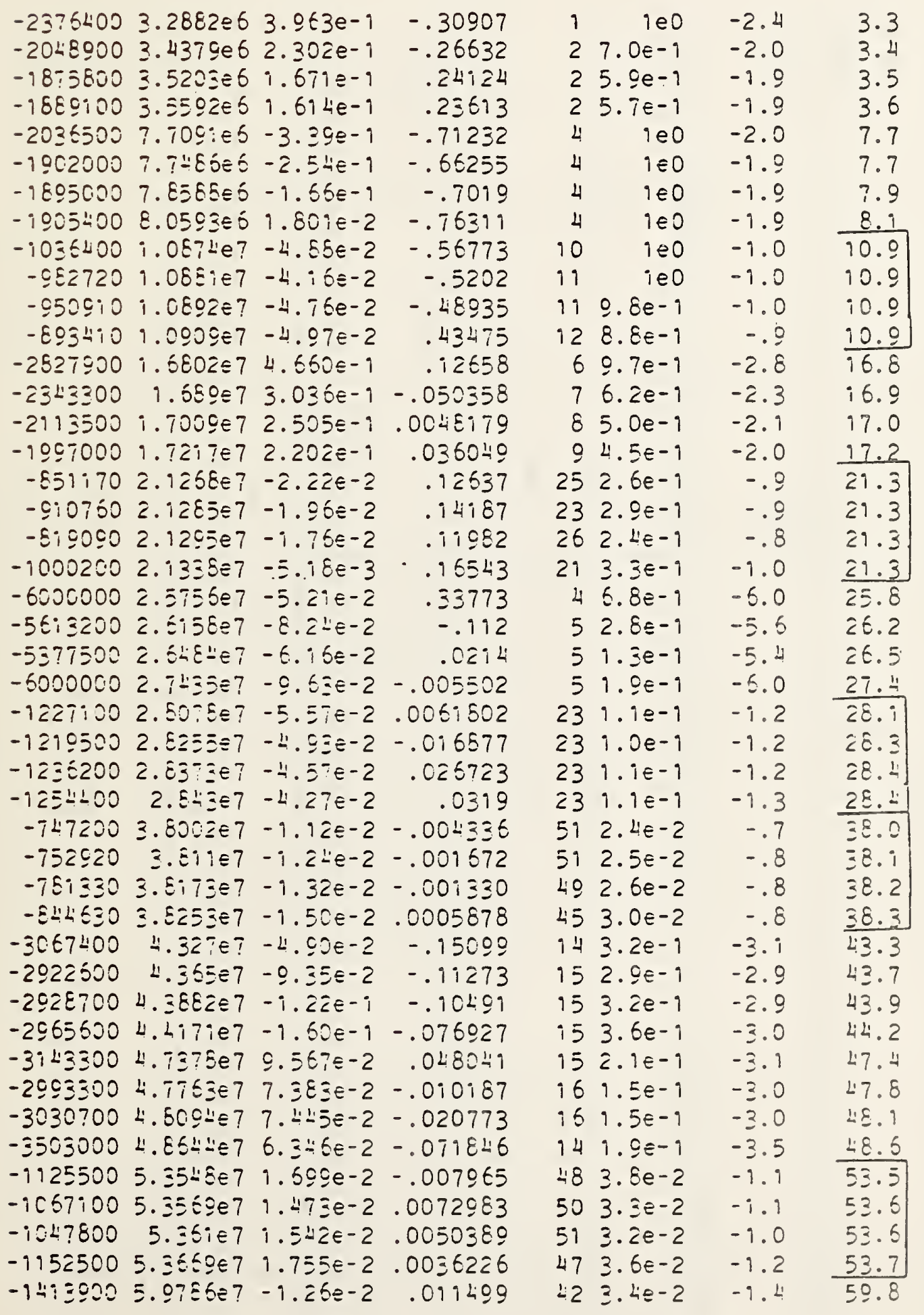




$$
\begin{array}{llll}
a & 0 & 0 & 0 \\
\sigma & \infty & \hat{N} & 0 \\
\sigma & \sigma & \sigma & \sigma \\
\times & \triangleright & \# & 0
\end{array}
$$

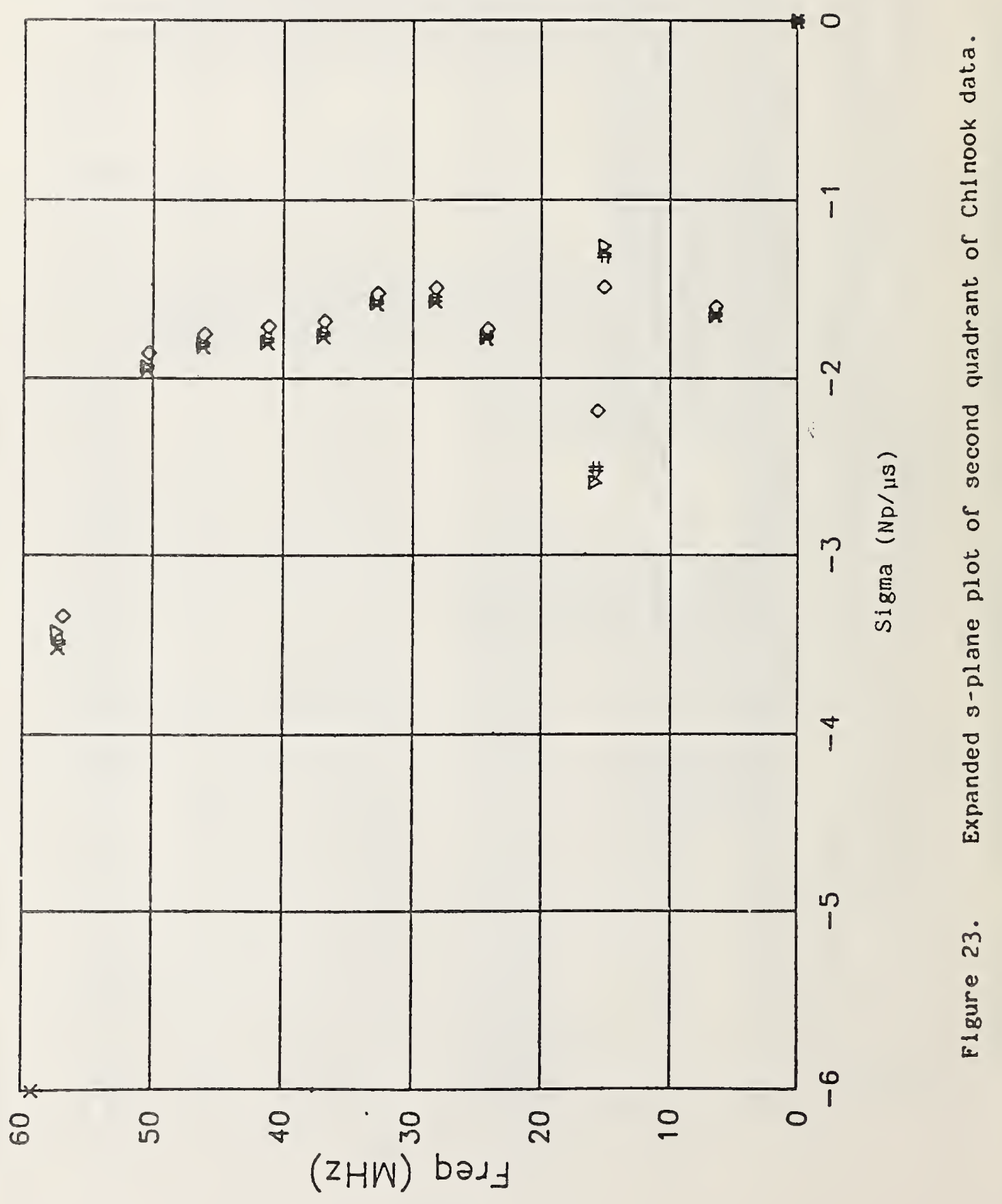


Table 4. Data from main-frame computer from Chinook signal. Table gives processed data of real and imaginary parts of complex frequency, real and imaginary parts of complex residue, Q, magnitude of residue, sigma and frequency.

$\begin{array}{ll}\text { Complex } & \text { ñesidue } \\ \text { Erequency } & \\ \text { Real Ime } 8 \text { ReEl Ineg }\end{array}$

$-15979006.4471 e 6-3.87 e-3.0045071$

$-16423006.4934 e 6-4.80 e-3.0058017$

$-i 6163006.5003 \in 6-6.58 e-3.007 \varepsilon \Sigma \varepsilon 5$

$-16477006.5106=6-5.10 \in-3.0062158$

$-1256100 \quad 1.5069 e 7 \quad 5.503 e-2$.064383

- $1282400 \quad 1.509 e 7 \quad 6.702 e-2 \quad .06559$

$\begin{array}{llll}-1306000 & 1.509 \xi \in 7 & 6.578 \epsilon-2 & .07104\end{array}$

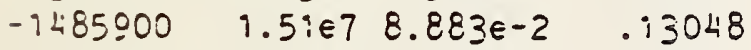

$-2 i \varepsilon 3000$ i.5555e7-9.52e-9 -.i454

$-25 i 09001.5754 e 7-1.37 \in-9-.082060$

-25582001.5787 e $7-9.44 e-9-.068 \div 55$

$-25874009.5860 \equiv 7-9.29 e-9-.071765$

$-17230002.4102 \in 7-9.74 e-2-.019879$

$-17660002.4 i 02 e 7-1.03 €-2-.010491$

$-17 T 5500 \quad 2.42 i \in 7-1.008-2-.005338$

$-17554002.1423507-1.05 E-2-.008 E 58$

$-\$ 4587002.8202 \epsilon 7-5.85 \epsilon-3-.038393$

$-95524002.8<6 \leqslant \leqslant 7-9.13 \varepsilon-2-.035554$

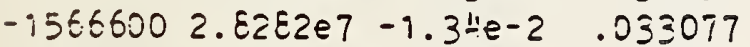

$\begin{array}{llll}-i 565100 & 2.8305 e 7 & -1.44 E-2 & .035984\end{array}$

$-15 i 02003.270 i e 73.612 E-2-.001461$

- $15711003.27 \varepsilon z e 7 \quad 3.619 e-2.0040745$

- $15826003.2 T$ TSET $3.592 e-2-.001453$

$-15747003.2 \varepsilon 22 e 7 \quad 3.57 \leqslant \epsilon-2-.005135$

$-9677000 \quad 3.67 \varepsilon \in 7-8.99 e-3 \quad-.0193$

$-9750500 \quad 3.687 \in 7-9.85 E-3-.005880$

$-1766000 \quad 3.689 e 7-1.03 e-2.000 i 536$

- $17567003.6999 \in 7-1.09 e-2.0032 i 81$

$-i 706000$ 4. $1 i 27 e 72.550 e-2.003 i \varepsilon 61$

$-1754 \Sigma 00$ 4. .12iइe7 2.4i5e-2 -.000400

- 1802500 4.123ze7 2.320e-2 .0033833

-1792000 L. $1262 e 7 \quad 2.312 e-2-.002306$

$-17502004.3 c \in 6 e 7-1.17 \epsilon-2-.017226$

-i811400 4.607ie7 -8.9eE-3 .020855

$-i 8251004.6097 \in 7 \quad-6.22 \epsilon-3 \quad .026146$

$\begin{array}{llll}-i 805000 \quad 4.612 e 7 & -5.30 £-3 \quad .022449\end{array}$

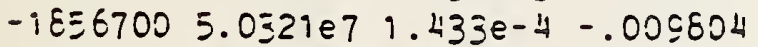

- $10 \$ 77005.0422 e 7-3.05 e-3-.006270$

$-1956 i 00 \quad 5.045 e 7-4.20 \epsilon-3-.000395$

$-i \leqq 341005.0475 \leqslant 7-5.3 i e-3-.001555$

- $3 \equiv \equiv 58005.6 \varepsilon \equiv 2 e 7 \quad i .432 \epsilon-3-.0143: 8$

$\begin{array}{lllll}-3 \div 83600 & 5.7177 e 7 & -6.6 i e-3 & -.010936\end{array}$

- $55 i 5700 \equiv .727 \varepsilon \in 7-3.96 e-4 \quad .00264 \varepsilon$

- $3 L 25000 \quad 5.7323 \in 7-\varepsilon .55 e-3.00 \varepsilon 1431$

$-6000000$
2

Mag

Res

Sitga Freo

$(\mathrm{NP} / \mathrm{NS}) \quad(\mathrm{M}: \mathrm{Z})$

\begin{tabular}{|c|c|c|c|}
\hline 4 & .012 & -9.6 & 6.4 \\
\hline 4 & .015 & -9.6 & 6.5 \\
\hline मे & .020 & -9.6 & 6.5 \\
\hline 4 & .096 & -9.6 & 5.5 \\
\hline 12 & .179 & $-1 \cdot 3$ & 95.9 \\
\hline 12 &.$~ 188$ & -9.3 & 15.9 \\
\hline 92 & .194 & -9.3 & $i 5 . i$ \\
\hline 10 & .396 & -9.5 & 15.1 \\
\hline 7 & .422 & -2.2 & 95.5 \\
\hline 6 & .319 & -2.5 & 15.6 \\
\hline 5 & .318 & -2.6 & $i 5.8$ \\
\hline 6 & .296 & -2.5 & 15.9 \\
\hline بل 9 & .042 & -9.7 & $24 . i$ \\
\hline 94 & .044 & -9.3 & 24.2 \\
\hline 94 & .042 & -9.8 & 211.2 \\
\hline 94 & .043 & -9.8 & $2 \div .2$ \\
\hline 19 & .078 & -9.5 & $\overline{28.2}$ \\
\hline is & .077 & -1.6 & 28.3 \\
\hline is & .079 & -9.5 & 28.3 \\
\hline 18 & .076 & -1.6 & 25.2 \\
\hline 22 & .072 & -1.5 & 32.7 \\
\hline 29 & .073 & -9.6 & 32.8 \\
\hline $2 i$ & .072 & -9.6 & 32.8 \\
\hline 21 & .073 & -9.6 & 22,8 \\
\hline 22 & .029 & -9.7 & $\overline{30.8}$ \\
\hline $2 i$ & .023 & -1.8 & 36.0 \\
\hline 29 & .029 & -9.8 & 36.9 \\
\hline 29 & .023 & -1.8 & $=5.0$ \\
\hline 24 & .053 & -1.7 & $49 . i$ \\
\hline 23 & .048 & -1.8 & 49.2 \\
\hline 23 & .047 & -9.8 & 49.2 \\
\hline 23 & .046 & -1.8 & 49.3 \\
\hline 26 & .042 & -9.8 & $\ddot{216.0}$ \\
\hline 25 & .045 & -9.8 & 46.9 \\
\hline 25 & .054 & -1.8 & $46 . i$ \\
\hline 26 & .047 & -1.8 & $\$ 6 . i$ \\
\hline 27 & .020 & -1.9 & 50.3 \\
\hline 26 & .015 & -9.9 & 50. \\
\hline 26 & .008 & -2.0 & 50.5 \\
\hline 25 & .014 & -1.9 & 50.5 \\
\hline$i 7$ & .029 & $-3 \cdot 3$ & 56.0 \\
\hline 96 & .025 & -3.5 & 57.2 \\
\hline 16 & .005 & -3.5 & 57.3 \\
\hline 97 & .024 & -3.4 & 57.3 \\
\hline io & 1.000 & -6.0 & 50.2 \\
\hline
\end{tabular}



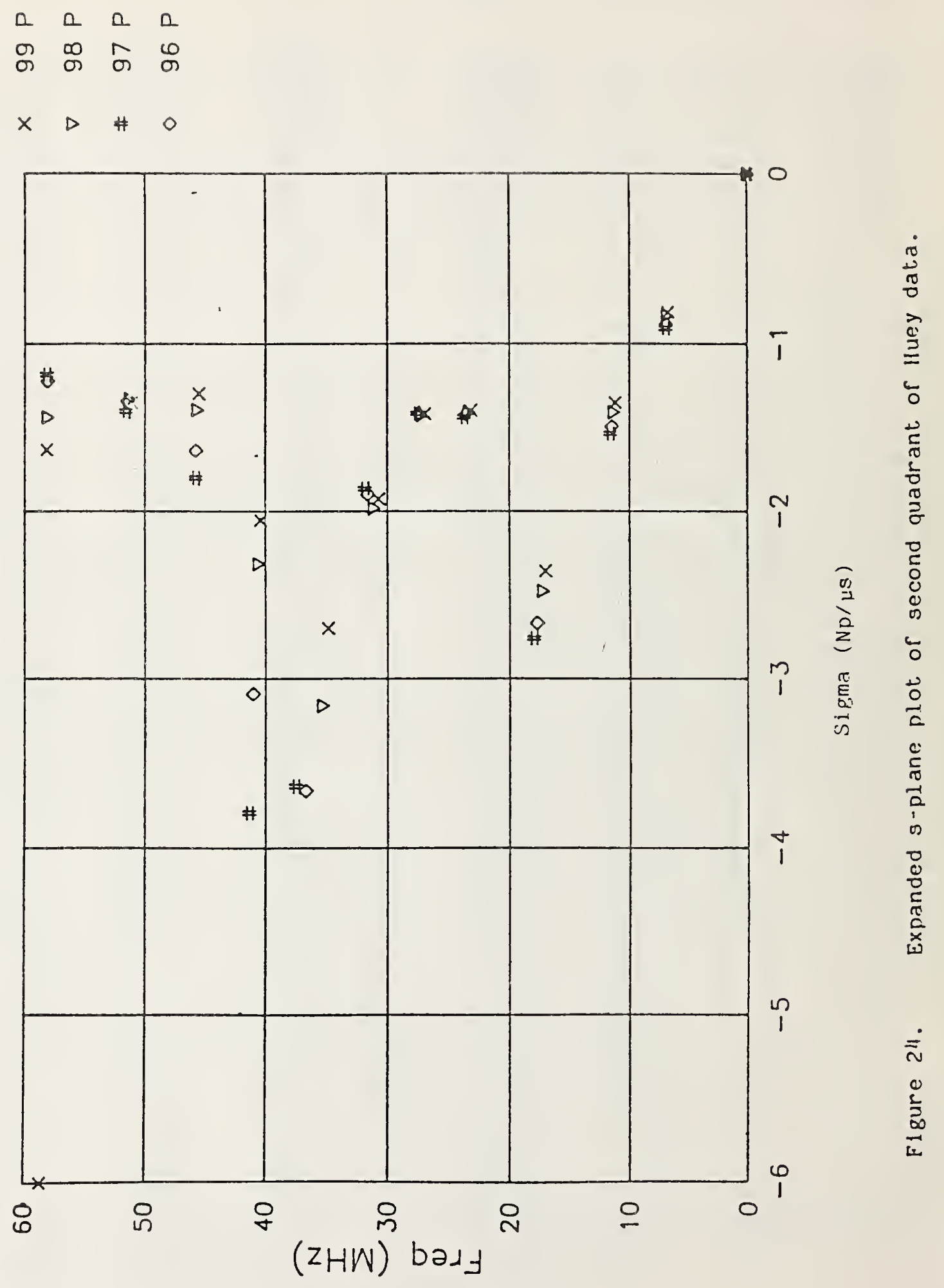
Table 5. Data from main-frame computer from Huey signal. Table gives processed data of real and imaginary parts of complex frequency. real and imaginary parts of complex residue, Q, magnitude of residue, sigma and frequency.

Complex
Frequency
feal ImeB $\quad$ Seà Imés
Q

Mag
Res

$-8134206.7849 e 6 \quad 3.009 e-3-.003374$

- $\varepsilon 331006.6465 e 6 \quad 3.084 \in-3.0035 i \equiv 3$

$-5 \$ 2380 \quad 6.5223 e 52.365 e-3 \quad-.00 \equiv 5 \equiv 5$

- $0.057606 .0605 e 6 \quad 1.520 e-3 \quad-.0021 E i$

$-9 \equiv 47300 \quad 1.1172 e 7 \quad 4.852 e-3.0052 i 62$

- iL0é200 i.iz66e7 5.393e-3 .00L2zzi

- $14 E 00009 . i 46 \varepsilon \in 7 \quad 6.448 \varepsilon-3.0024664$

$-i 5355009 . i 556 \in 7 \quad 5.755 e-3.00374 i 5$

- $\equiv \leqslant 91009.7049 \in ?-9.60 E-2$.00Eミ307

$\begin{array}{lll}-2479700 & 1.7263 E 7 & -1.606-2 \quad .010923\end{array}$

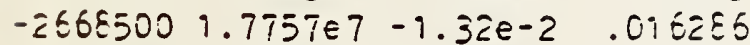

$-2757 i 00 \quad 1.8001 \in 7-i .25 e-2 \quad .024 i 95$

$\begin{array}{lll}-1393900 & 2.3132 e 7-2.22 e-3 & -.010414\end{array}$

$-91012002.3203 e 7-6.25 e-3-.0107 \equiv 9$

-iL15000 2.3549e7-i.25e-2 -.00Ei 14

- $1 H \equiv 22002.3657 e 7-i .4 g \in-2-.002872$

- $94 i 5700 \quad 2.6937 \in 7 \quad 0.025 e-3 \quad .017273$

- i $125200 \quad 2.7933 \mathrm{e} 7 \quad$ 1.7ELe-2 .0i 1905

- $i \leqslant 057002.7425 e 7 \quad 2.3148 e-2 \cdot-.002476$

$-9490500 \quad 2.7554 \leqslant 7 \quad 2.298 e-2-.0067 i 0$

- $192 \div 9003.0722 e 7-2.44 \in-2 \quad .003 i 6 \varepsilon 3$

$-1982800 \quad 3.107 € 7-2.52 \epsilon-2 \quad .017508$

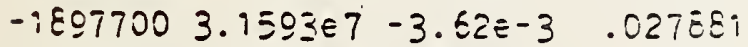

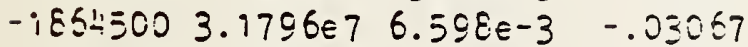

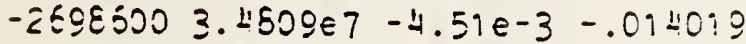

-5i59400 3.5228 e $7-1.60 e-2 \quad .0 i 588$

- $\$ 650100 \quad 3.6637 \in 7-3.03 \epsilon-2-.092 \varepsilon \equiv 2$

- $5532400 \quad 3.7473 e 7-7.8 i e-3 \quad .05266$

-205i900 म.03E3e7 - $8.89 \in-3-.00 E-21$

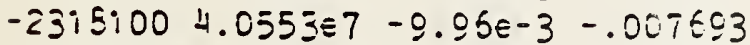

- 3058100 4. $102 i \in 7-1.08 E-2-.010761$

- $\$ 585500$ 4.131e7 $-6.05 E-2$.00E6777

- 1292000 4.5山5山E $-7.89 e-3-.007070$

$-i 5905004.5562 e 7-8.74 \in-3-.005597$

-1758700 म. $5701 e 7-1.13 e-2-.005 \varepsilon 95$

-i6 $\$ \equiv 2004.5793 \in 7-7.06 e-3-.005308$

$-i \Sigma \vdots 7500 \equiv .1 i 22 \epsilon 7-4.75 \epsilon-3-.0104 \equiv 7$

$-1 \equiv 2=0005.1264 \in 7-6.77 e-3-.005018$

$-15 \Sigma 74005.1476 e 7-5.85 e-3-.004 \equiv 27$

- i $507900 \quad 5.1548 \leqslant 7-7.05 e-3.0024004$

$-14 \equiv 57005.5073 e 7-4.25 \epsilon-3.005171-9$

$-12 i \equiv 5005.8055 e 7-i .10 e-3 \quad .001540$

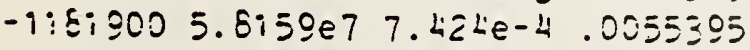

- i६E=500 $5.8 i 6 e 7-6.12 e-3.0053045$

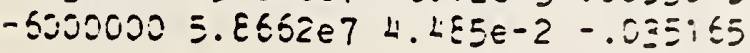

.010

$\varepsilon \quad .009$

$8 \quad .009$

8.006

6.094

8.014

8.094

8.094

7.053

$7 \quad .040$

7.042

7.054

$i 7 \quad .029$

17.023

$17.0 \leqq 0$

17.030

io .040

$19 \quad .043$

ig .047

20.016

$.6 \quad .049$

i5 .058

$.7 \quad .056$

$17 \quad .063$

$13 \quad .029$

$1 i \quad .048$

10.065

i0 . 905

$20 \quad .022$

17.025

13.045

i 9.929

इ5 .029

33

25

28

33

29

38

37

40

48

19

35

10
Sigma EreC

(Np/us) ( $\mathrm{N}-\mathrm{i} z$ )

$-.8$

6.8

$-.8$

6.8

$-.9$

6.9

$-.9$

7.0

$-i .3$

$-i .1$

$-9.5$

$-1.5$

$-2.4$

$-2.5$

$-2.7$

$-2.8$

$-i .4$

$-9.4$

- 1.4

$-i .4$

$-9.4$

$-9.4$

$-9.4$

$-9.4$

$-9.0$

$-2.0$

$-9.0$

$-1.9$

$-2.7$

$-\overline{3} .2$

$-3.7$

$-3.5$

$-2.9$

$-2.3$

$-3.1$

$-3.8$

$-1.3$

$-1.4$

$-1.8$

$-9.5$

$-1.3$

$-1.3$

$-1.3$

$-1.4$

$-1.4$

$-1.2$

$-1.2$

$-1.6$

$-6.0$
99.2

11.3

19.5

91.5

17.0

17.3

17.8

$\frac{i 8.0}{23 . i}$

¿三.

23.5

$\frac{2^{2} .7}{26.9}$

27.1

27.

$\frac{27.6}{30.7}$

三i.

3.6

30.5

三.

इ⿰氵工 2

36.6

37.5

10.

10.5

49.0

$\frac{4 i \cdot 3}{-5.3}$

$4 三 .6$

15.7

$\frac{\not 5.7}{51.1}$

91.3

51.

$\frac{51 .}{56 .}$

E.

Ė.

$\equiv \varepsilon .2$

55.7 


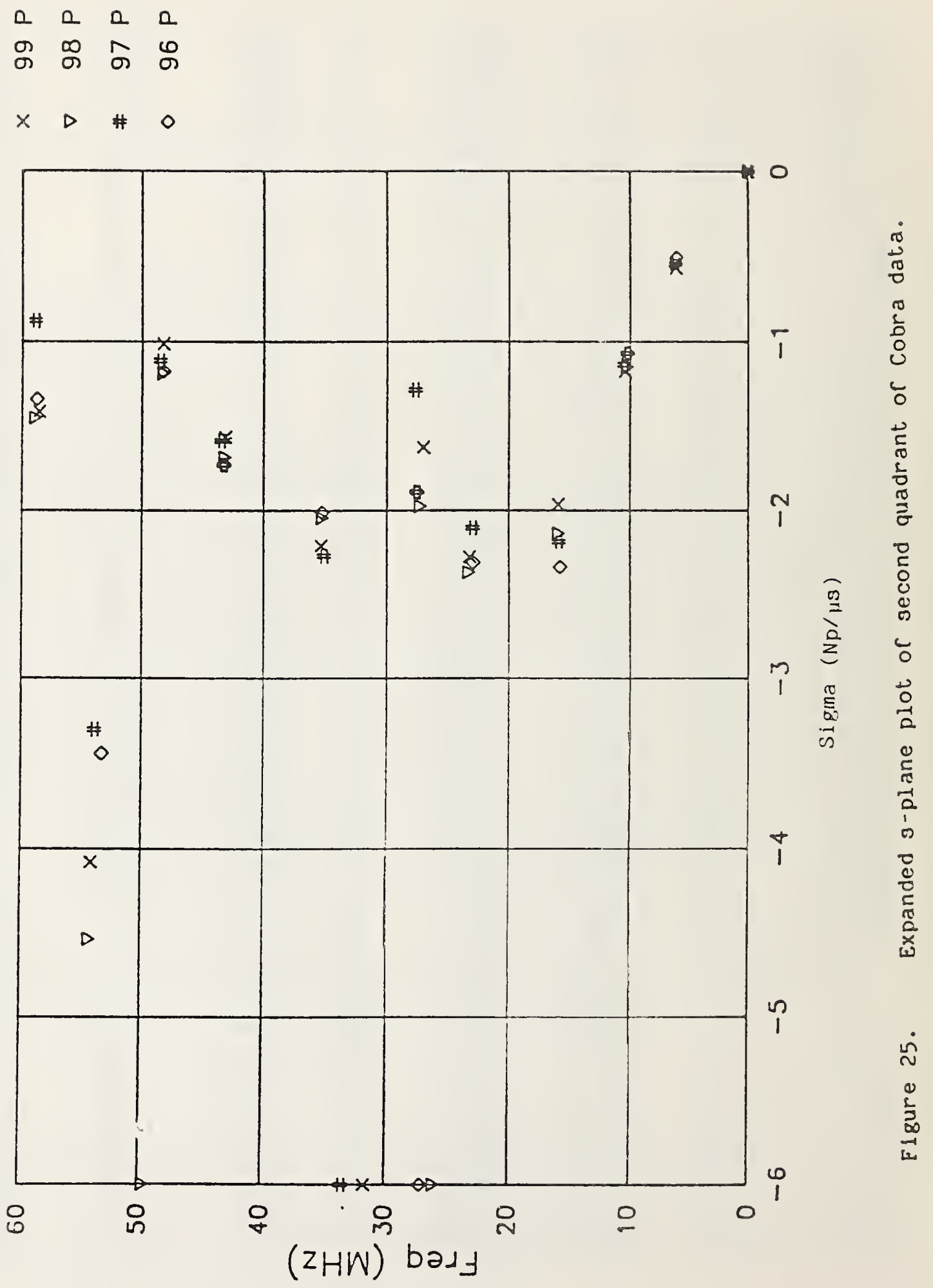


Table 6. Data from main-frame computer of Cobra signal. Table gives processed data of real and imaginary parts of complex frequency, real and Imaginary parts of complex residue, $Q$, magnitude of residue, sigma and frocuency.

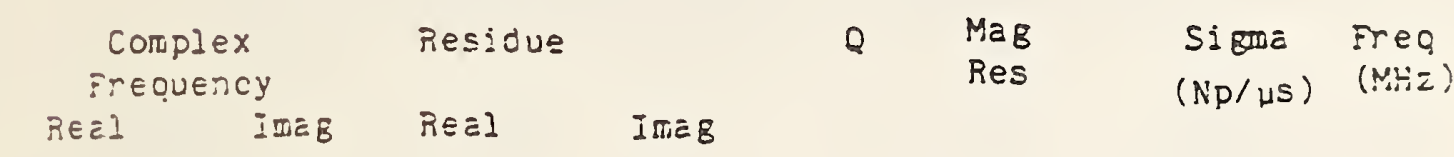

\begin{tabular}{|c|c|c|c|c|c|c|c|}
\hline-506080 & $6.07 c 5 \leqslant 6$ & $-4.75 e-3$ & -.052238 & 12 & .105 & -.5 & 6.1 \\
\hline-540610 & 6.087526 & $-5.04 e-3$ & .055038 & 11 & .111 & -.5 & 6.1 \\
\hline-567150 & 6. 1isie6 & $-0.24 \in-3$ & .057526 & 11 & .197 & -.6 & 6.1 \\
\hline-544700 & $6.1322 E 6$ & $-0.90 \in-3$ & -.054790 & 19 & .999. & -.5 & 6.1 \\
\hline - 1067700 & $1.0201 \in \mathbb{7}$ & $4.656 \varepsilon-2$ & .034901 & 10 & .915 & -1.1 & 10.2 \\
\hline$-194 \varepsilon \equiv 00$ & 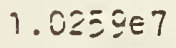 & $4.705 e-2$ & -.049524 & Q & .126 & -1.1 & 10.3 \\
\hline$-1974 \leq 00$ & $1.02 ; \vdots \leqslant 7$ & $3.848 \epsilon-2$ & -.051846 & 9 & .120 & $-i .2$ & 10.4 \\
\hline$-113 \div 500$ & $1.0 \div 53 \in 7$ & $3.715 e-2$ & .048735 & 9 & .123 & -1.1 & 10.5 \\
\hline-2341000 & $1.57 \div 8=7$ & E. $55 i \in-2$ & -.090385 & 7 & .250 & $-2 \cdot 3$ & $i \equiv .7$ \\
\hline$-2 i \varepsilon \varepsilon 500$ & 1. $\equiv \varepsilon \div 6 \subseteq 7$ & $7.685 e-2$ & -.075234 & 7 & .215 & -2.2 & $i \equiv .8$ \\
\hline - 10E乏60J & $1.58 \varepsilon \varepsilon \varepsilon=7$ & $6.27 z \varepsilon-2$ & .057383 & 8 & .170 & -2.0 & 15.0 \\
\hline$-2135 \leq 00$ & $1.5 E 55 \leqslant 7$ & $8.023 \epsilon-2$ & .057665 & 7 & .210 & $-2 . i$ & 15.9 \\
\hline$-23 ; 4000$ & 2. $2 c^{0}: \leqslant 7$ & $-7.3 i e-4$ & -.19484 & 10 & .200 & -2.3 & 22.9 \\
\hline$-2 i 0 \leqq 000$ & 2. ¿છ: $9 \in 7$ & $-1.58 \epsilon-4$ & -.9497 & 19 & .200 & -2.1 & 22.9 \\
\hline$-2<80000$ & 2. $\vdots i \leqq 5 \leqq 7$ & $-5.07 e-2$ & -.17953 & $: 0$ & .373 & -2.3 & 23.2 \\
\hline -237ンー0ல & こ. ここテうе & $-7.6 \subseteq \varepsilon-2$ & $-.201 E 6$ & 10 & .432 & -2.4 & $=3.3$ \\
\hline-6000000 & 2. $\equiv \sum \subseteq \sum \varepsilon 7$ & $5.93 \bar{x}$ & .47865 & 4 & 1.000 & -6.0 & 26.0 \\
\hline$-16 z \div 500$ & 2. ธQ๑Eڤ7 & $-2.51-\varepsilon-2$ & -.025499 & 97 & .072 & -1.5 & 27.0 \\
\hline-6000000 & 2.7iole? & $3.560 E-1$ & .3277 & 3 & $.0 \in g$ & -6.0 & $27 . i$ \\
\hline$-997+400$ & 2. $725 i 27$ & $-5.5 i \in-2$ & -.025256 & 94 & .121 & -2.0 & 27.3 \\
\hline-1800500 & 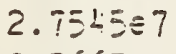 & $-5.2\} \in-2$ & $-.01<673$ & i5 & .107 & -1.9 & 27.5 \\
\hline$-: \geq \varepsilon १$ ¿ & 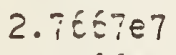 & $-1.65 \leqslant-2$ & .0006369 & 22 & .033 & $-1 \cdot 3$ & 27.7 \\
\hline-5000000 & $3 . ; 66 € 7$ & 4. $2 \div 0 \leqslant-9$ & -.96455 & 5 & .990 & -6.0 & 31.7 \\
\hline-5000200 & $3.34 \div 6 \in 7$ & $3.8 \varepsilon 5 \varepsilon-1$ & $-.069 \equiv 92$ & 6 & .778 & -6.0 & 33.4 \\
\hline$-227 \div 200$ & 3.5015507 & $-9.3 i E-2$ & $.08 \equiv 202$ & 15 & $.2 \equiv 2$ & -2.3 & $\vdots \equiv .0$ \\
\hline$-20 \div \div 500$ & 3. $三 20$ se? & $-5.36 \varepsilon-2$ & .07178 & 17 & .179 & -2.0 & 35.2 \\
\hline$-20 i 0200$ & $\underline{3.52 z \delta \in 7}$ & $-4.75 \equiv-2$ & .074729 & 18 & .977 & -2.0 & $\$ 5.2$ \\
\hline$-22 i<600$ & ミ.5ะๆ山@7 & $-5.40 e-2$ & $.0937 i 4$ & i6 & .216 & $-\hat{z} .2$ & 35.3 \\
\hline$-156 \equiv i 00$ & $4.9 ; 07 e 7$ & $-2.93 e-2$ & .015519 & 28 & .065 & -1.6 & $43 . i$ \\
\hline$-15 \varepsilon \equiv 000$ & แ. & $-3.2 z \epsilon-2$ & .021537 & 25 & .078 & -1.7 & 43.2 \\
\hline-1728500 & 4. $\overline{3} 2 \overline{2 e} 7$ & $-3.0 \leq e-2$ & .023753 & 25 & .077 & -1.7 & 43.2 \\
\hline - : & 4. $\equiv=9787$ & $-2 . \overline{3} i \in-2$ & .027017 & 27 & .071 & -1.5 & 43.4 \\
\hline$-1972 i 00$ & 4. $520 ; \mathrm{e} 7$ & $-5.5 \bar{x} \in-3$ & .01317 & 49 & .028 & -1.2 & 48.2 \\
\hline$-10 i 0300$ & 4. .ระi & $-7.01 \varepsilon-3$ & .010554 & 1.3 & .025 & -1.0 & 48.2 \\
\hline$-11 \varepsilon \equiv 300$ & 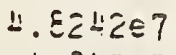 & $-7.60 \leqslant-3$ & .013736 & 49 & .031 & -1.2 & 48.2 \\
\hline-19053 & 4. E4 & $-8.02 e-4$ & -.015686 & 44 & .031 & -1.1 & $48 . \equiv$ \\
\hline-6000000 & 4. Ei Se 7 & $-2.5 ? e-1$ & .97015 & 8 & .605 & -6.0 & 19.5 \\
\hline-3455500 & $5.3=78 \mathrm{e} 7$ & $5 . \varepsilon \equiv 4 \varepsilon-2$ & -.034299 & $i 5$ & .135 & -3.4 & 53.3 \\
\hline-3200200 & 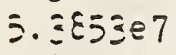 & 4. $3 \subseteq \varepsilon \leqslant-2$ & -.042452 & 16 & .122 & -3.3 & 53.9 \\
\hline-4076000 & 5.1902 & $3.394 \in-2$ & $-.077 i 63$ & 13 &.$i 63$ & $-4 . i$ & 54.1 \\
\hline-4520000 & $\Xi .42 \equiv 8$ & 5.i $\vdots \vdots e-2$ & -.090219 & 12 & .224 & -11.5 & $=4.2$ \\
\hline-1419400 & $5 . \varepsilon \equiv 27 e 7$ & $-4.39 \in-3$ & .0033204 & $\therefore 1$ & .011 & -1.4 & 58.5 \\
\hline-1336000 & $5.87-8 e 7$ & $-7.5-e-4$ & .0052572 & 44 & .011 & -1.3 & 58.7 \\
\hline & $5 . £ £ 5$ & $-8.65 \epsilon-4$ & .0037235 & 57 & .008 & -.9 & 58.9 \\
\hline & $\Xi . E \varepsilon \xi$ & $-5.60 e-4$ & .00521007 & 11 & $.5 i 1$ & $-i .4$ & $5 \varepsilon . \vartheta$ \\
\hline
\end{tabular}




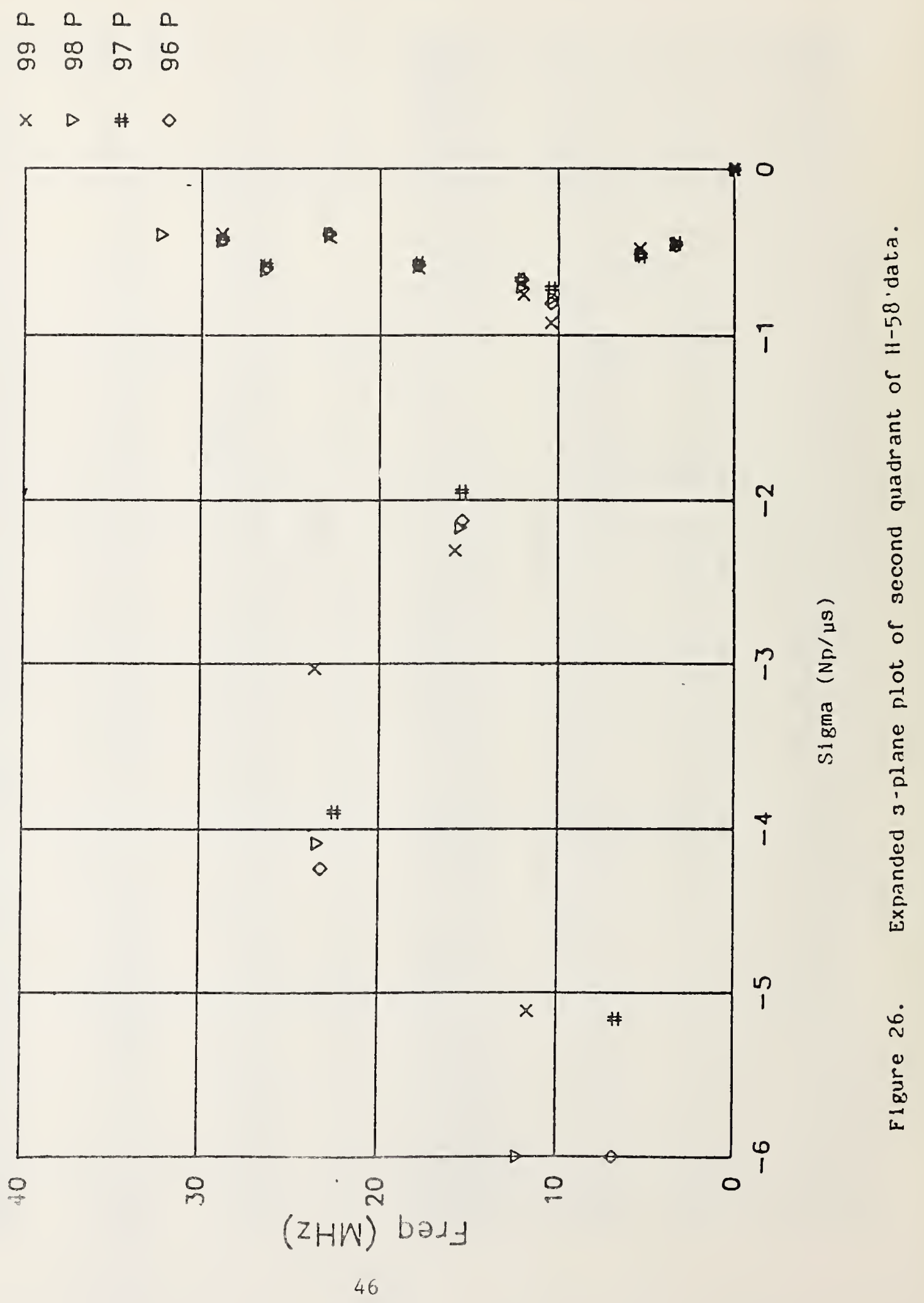


Table 7. Data from main-frame computer of H-58 signal. Table gives processed data of real and imaginary parts of complex frequency, real and imaginary parts of complex residue, $Q$, magnitude of residue, sigma and frequency.

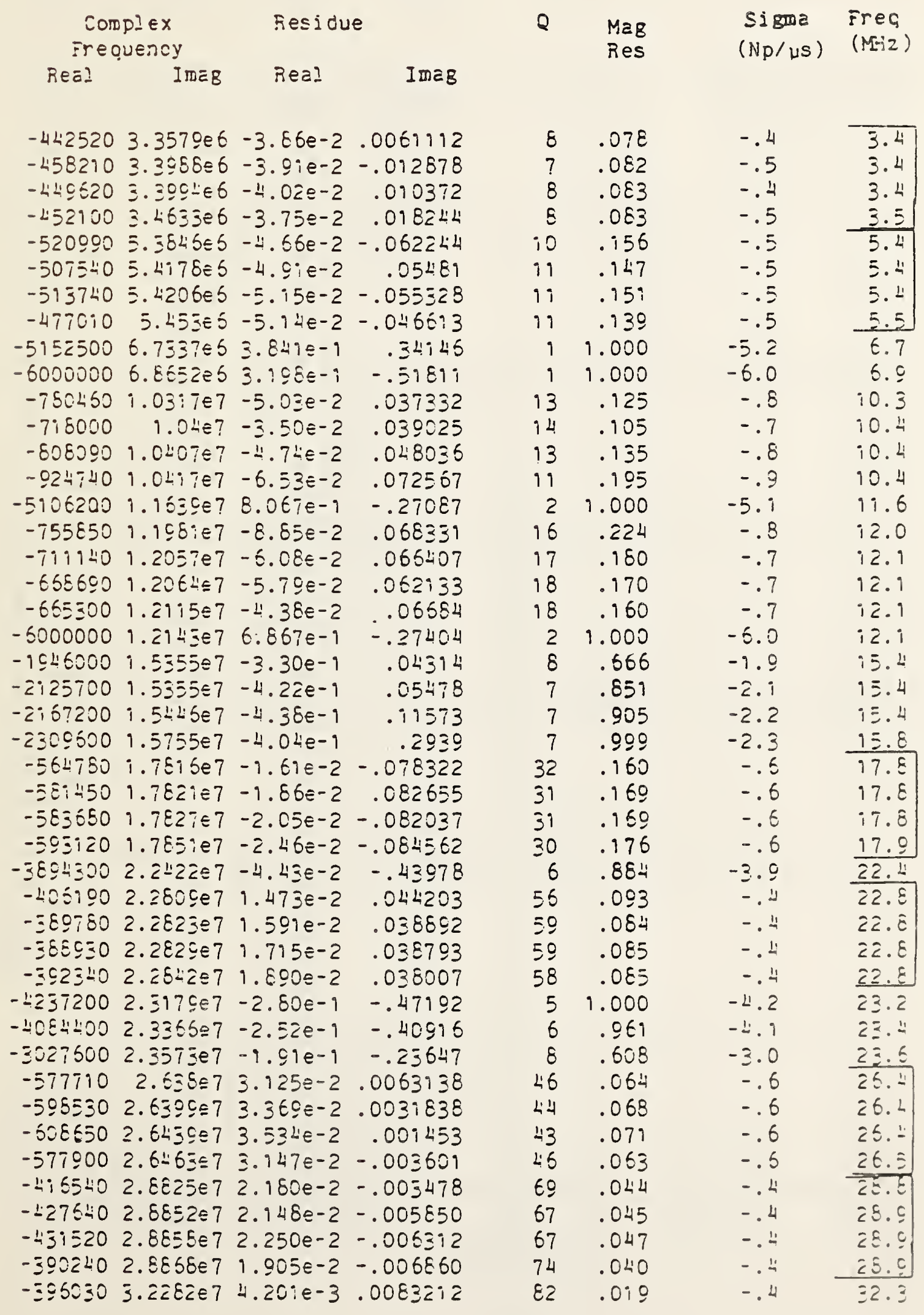




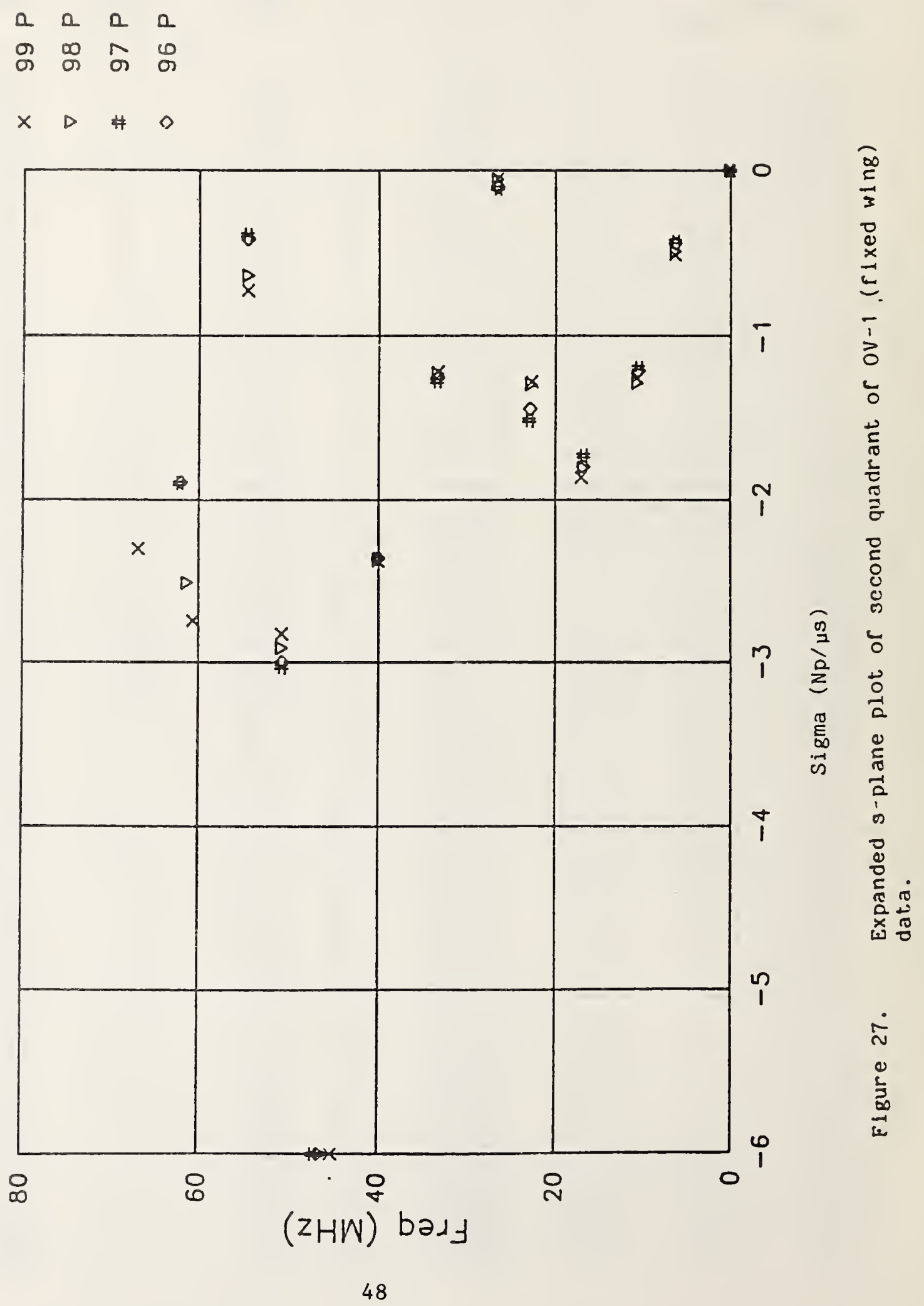


Table 8. Data from main-frame computer of ov-1 (fixed wing) signal. Table gives processed data of real and imaginary parts of complex frequency, real and imaginary parts of complex residue, $Q$, magnitude of residue, sigma and frequency.

$\begin{array}{ll}\text { Compiex } & \text { Residue } \\ \text { Freouency } & \\ \text { Real Imab Real Imag }\end{array}$

$-4290406.2423 e 6-3.46 e-2-.028586$

$-4433606.2452 e 6-3.56 e-2-.029179$

$-454 T i 0$ 6. $2504 \leqslant 6-3.05 e-2-.031785$

$-5 i \equiv 070$ 6.2772e6 -4.29e-2 -.031909

$-190 ;=00$ i.04ibe? 1.709e-2-.070894

$-12 i 6 i 00 \quad 1.05 i \in 7 \quad 1.532 \in-2 \quad .073028$

-1282300 i. $06 i j e 75.675 e-3-.070524$

- $12 \equiv 6200$ 1.0бEइE7 - $1.20 E-3-.0763 \equiv 1$

$-97 \overline{20000}$ i. $6703 \pm 7-1.57 \mathrm{e}-2-.009756$

$-i 505300$ i. $671907-2.02 e-2 \quad .013 \overline{i 3}$

$-9 T E E \equiv 00$ i. $67 \equiv E E 7-1.67 e-2-.012 i 75$

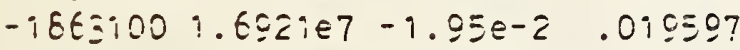

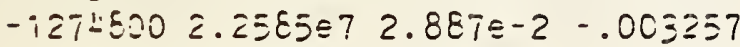

$\begin{array}{llll}-120 i 500 & 2.2705 e 7 & 2.875 e-2 & -.007253\end{array}$

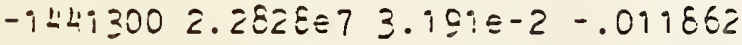

- 1510000 z. $28 E 5 e 7 \quad 3.25 i e-z \quad .014516$

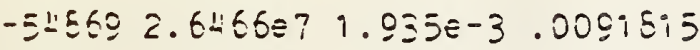

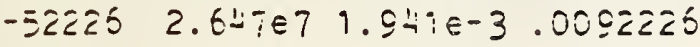

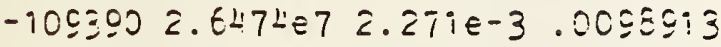

-i00720 z.6477e7 2.3i6e-3.0098i75

$-1216000 \equiv . \equiv 164 e 7-2.25 \epsilon-2.001<\varepsilon \equiv \equiv$

- $1226 L 00 \xi .3225 e 7-2.2 \varepsilon \in-2.00290: 3$

$-i z 1100003.3<E E \leqslant 7 \quad-2.30=-2.0049 E 98$

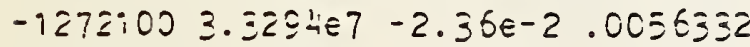

-2360200 z.09i6e7-1.30e-1 .20753

-23EL900 j.ggLie7 -i.32e-9 .20778

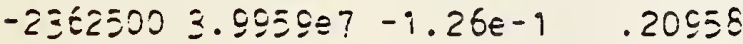

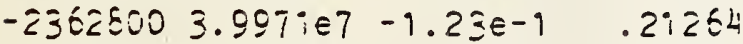

$-6000000 L .50 L 5 E 7-1.17 \epsilon-i \quad-. E \equiv 0 \Sigma 5$

$-60000004.6005 \leqslant 7-3.00 e-1 \quad-.05872$

$-60000004.65 \equiv 5 e 7-4.17 e-1-1.0208$

$-60000004.608657 \quad-5.42 e-1 \quad-1.027 i$

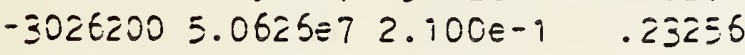

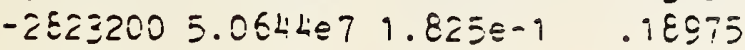

$-20955005.0645 E 72.086 e-9 \quad .22509$

$-20 i 19005.0585=72.068 \in-i \quad .20 i 3 \div$

- $639250 \equiv .4525 e 7$ 6.040e-3 -.00\$-05

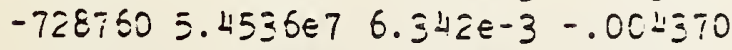

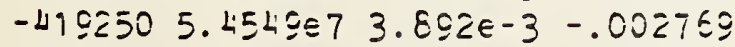

- $\$ 5 \subseteq \$ 00 \quad 5.2573 \in 7 \quad 3.5 \subseteq 6 \in-3-.0020147$

-274L900 6.04Eie7 5.005e-4 -.0iELL1

$-251<3006.1981 e 7-9.08 E-2-.018 \div 73$

-iEgiico $6 . i £ 23 e 7-1.37 e-2-.00 L \varepsilon \leqslant 3$

$-i \varepsilon c i g 00 \quad 6.2058 \varepsilon 7-1.59 e-2-.003022$

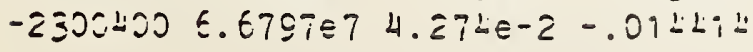

Q Mag
Res

9

13

12

0$$
\text { 9 }
$$

8

9

io

9

象

1

i5

is

16

is

200

200

200

200

27

27

27

25

i7

17

17

17

$$
8
$$

$$
8
$$

$$
\begin{aligned}
& 8 \\
& 8
\end{aligned}
$$

6

17

i 8

17

17

85

75

150

120

22

24

29

\author{
Sigma Freo \\ $(\mathrm{Np} / \mathrm{HS}) \quad(\mathrm{M}: \mathrm{Z})$
}

$-.4$

6.2

$-.4$

6.2

$-.5$

$-.5$

$-9.2$

$-1.2$

$-1.3$

$-1.3$

$-1.7$

$-1.8$

$-9.8$

$-1.9$

$-1.3$

$-1.3$

$-9.4$

$-i .5$

$-.1$

$-.1$

$-.1$

$-.1$

$-i .2$

$-1.2$

$-1.2$

$-i .3$

$-2.4$

$-2.4$

$-2.4$

$-2.4$

$-6.0$

$-6.0$

$-6.0$

$-6.0$

$-3.0$

$-2.8$

$-3.0$

$-2.9$

$-.5$

$-.7$

$-.4$

$-.4$

$-2.7$

$-2.5$

$-1.9$

$-1.9$

$-2.3$
6.3

$\frac{6.3}{i 0.5}$

10.5

10.6

$\frac{i 0.7}{i 5.7}$

16.7

$i 5.8$

$\frac{15.9}{22.5}$

22.7

2द. E

22.9

26.5

26.5

$\frac{26.5}{33.2}$

इ3.2

इ3. 3

$\frac{23.3}{30.5}$

39.9

10.0

$\because 0.0$

$L 5.0$

15.0

LE. 6

$\frac{-7.0}{50.6}$

E0.6

50.6

$\equiv 0.7$

$\equiv .5$

三..

$= \pm .6$

50.5

Ei. 2

Ei.

E2. 1

65.8 
Table 3. List of Aircraft, natural frequencies, and approximate $Q$. * Aliased frequencies ( $f$ alse).

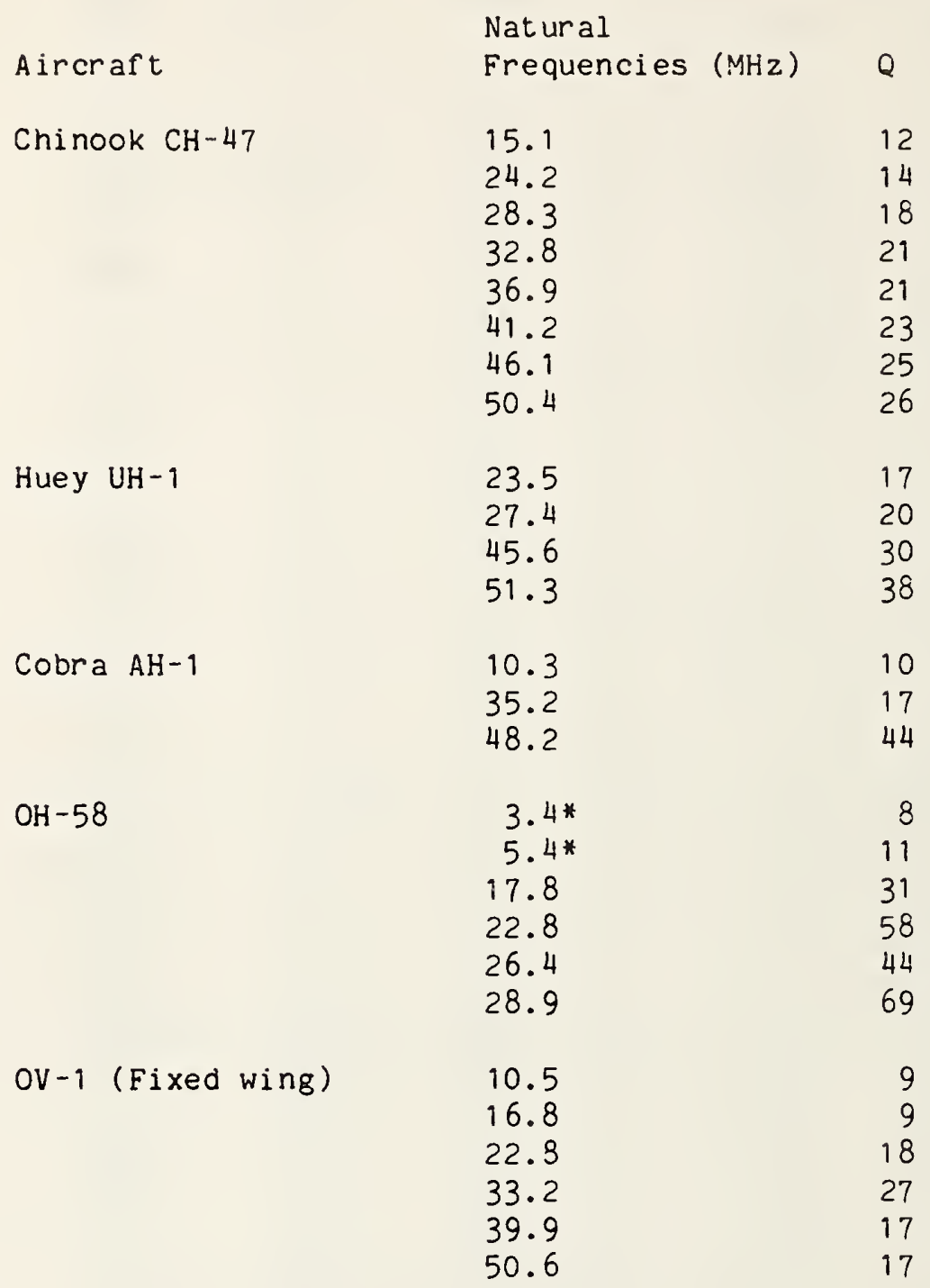


NBS-IIAA IREV. 2-80)

U.S. OEPT. OF COMM.

BIBLIOGRAPHIC DATA

SHEET (See in struction s)

1. PUBLICATION OR REPORT NO.

NBSIR $87-3077$

4. TITLE AND SUBTITLE

Time-Domain System for Identification of the Natural Resonant Frequencies of

Aircraft Relevant to Electromagnetic Compatibility Testing

5. $\operatorname{AUTHOR}(S)$

John W. Adams, Arthur R. Ondrejka, and Herbert W. Medley

6. PERFORMING ORGANIZATION (If joint or other than NBS, see instructions)

NATIONAL BUREAU OF STANDARDS

DEPARTMENT OF COMMERCE

WASHINGTON, D.C. 20234

9. SPONSORING ORGANIZATIOHA NAME AND COMPLETE ADDRESS (Straet, City, StOTe, ZIF)

7. ContracdGrant No.

8. Type of Report \& Period Covered

U.S. Army Aviation Systems Command

St. Louis, Missouri 63120

10. SUPPLEMENTARY NOTES

Document describes a computer program; SF-185, FIPS Software Summary, is attached.

11. ABSTRACT (A 200-word or less factual summary of most significant information. If document includes a significant bibliography or literature survey, mention it here)

A method of measuring the natural resonant frequencies of a structure is described. The measurement involves irradiating this structure, in this case a helicopter, with an impulsive electromagnetic (EM) field and receiving the echo reflected from the helicopter. Resonances are identified by using a mathematical algorithm based on Prony's method to operate on the digitized reflected signal. The measurement system consists of special TEM horns, pulse generators, a time-domain system, and prony's algorithm. The frequency range covered is 5 megahertz to 250 megahertz; this range is determined by antenna and circuit characteristics.

The measurement system is demonstrated, and measured data from several different helicopters are presented in different forms. These different forms are needed to determine which of the resonant frequencies are real and which are false. The false frequencies are byproducts of Prony's algorithm.

12. KEY WORDS (Six to twelve entries; alphabetical order; capitalize only proper names; and separate key words ty semicolons) avalanche step generator; natural resonant frequencies; Prony's method; TEM horn antennas; time-domain measurement system.

13. AVAILABILITY

QXnlimited

$\square$ For Official Distribution. Do Not Release to NTIS

$\square$ Order From Superintendent of Documents, U.S. Government Printing Office, Washington, D.C. 20402.

X Order From National Technical Information Service (NTIS), Springfield, VA. 2216I
14. NO. OF

PRINTED PAGES

56

15. Price 


